\title{
Quality control and processing of nascent RNA profiling data
}

Jason P. Smith ${ }^{1,4}$, Arun B. Dutta ${ }^{4}$, Kizhakke Mattada Sathyan ${ }^{4}$, Michael J. Guertin ${ }^{1,4, 凶}$, and Nathan C. Sheffield $1,2,3,4, \bowtie$

\footnotetext{
${ }^{1}$ Center for Public Health Genomics, University of Virginia

${ }^{2}$ Department of Public Health Sciences, University of Virginia

${ }^{3}$ Department of Biomedical Engineering, University of Virginia

${ }^{4}$ Department of Biochemistry and Molecular Genetics, University of Virginia

$\triangle$ Correspondence: guertin@virginia.edu

$\bowtie$ Correspondence: nsheffield@virginia.edu
}

Experiments that profile nascent RNA are growing in popularity; however, there is no standard analysis pipeline to uniformly process the data and assess quality. Here, we introduce PEPPRO, a comprehensive, scalable workflow for GRO-seq, PRO-seq, and ChROseq data. PEPPRO produces uniform processed output files for downstream analysis, including alignment files, signal tracks, and count matrices. Furthermore, PEPPRO simplifies downstream analysis by using a standard project definition format which can be read using metadata APIs in $\mathrm{R}$ and Python. For quality control, PEPPRO provides several novel statistics and plots, including assessments of adapter abundance, RNA integrity, library complexity, nascent RNA purity, and run-on efficiency. PEPPRO is restartable and fault-tolerant, records copious logs, and provides a web-based project report for navigating results. It can be run on local hardware or using any cluster resource manager, using either native software or a provided modular Linux container environment. PEPPRO is thus a robust and portable first step for genomic nascent RNA analysis.

Availability: BSD2-licensed code and documentation: https://peppro.databio.org.

\section{Background}

Steady-state transcription levels are commonly measured by RNA-seq, but there are many advantages to quantifying nascent RNA transcripts: First, it measures the transcription process directly, whereas steady-state mRNA levels reflect the balance of mRNA accumulation and turnover. Second, nascent RNA profiling measures not only RNA polymerase occupancy, but also orientation, and can be used to determine pausing and accumulation within any genomic feature. While RNAseq can measure transcript orientation, this depends specifically on how the RNA-seq library preparation step was performed. In contrast, orientation is inherent in PRO-seq library construction and therefore, all PRO-seq libraries measure transcript orientation by default. Third, nascent RNA profiling measures unstable transcripts, which can be used to infer regulatory element activity and identify promoters and enhancers de novo by detecting bidirectional transcription and clustered transcription start sites (TSSs) ${ }^{1,2}$. These advantages have led to widespread adoption of global run-on (GROseq), precision run-on (PRO-seq), and chromatin run-on (ChRO-seq) experiments ${ }^{3-5}$. With increasing data production, we require analysis pipelines for these data types. While tools are available for downstream analysis, such as to identify novel transcriptional units and bidirectionally transcribed regulatory elements ${ }^{1,6-10}$, there is no comprehensive, unified approach to initial sample processing and quality control.

Here, we introduce PEPPRO, an analysis pipeline for uniform initial sample processing and novel quality control metrics. PEPPRO features include: 1) a serial alignment approach to remove ribosomal DNA reads; 2) nascent transcription-specific quality control outputs; and 3) a modular setup that is easily customizable, allowing modification of individual command settings or even swapping software components by editing humanreadable configuration files. PEPPRO is compatible with the Portable Encapsulated Projects (PEP) format, which defines a common project metadata description, facilitating interoperability. PEPPRO can be easily deployed across multiple samples either locally or via any cluster resource manager, and we also produced a computing environment with all the command-line tools required to run PEPPRO using either docker or singularity with the bulker multi-container environment manager ${ }^{11}$. Thus, PEPPRO provides a unified, cross-platform pipeline for nascent RNA profiling projects. 


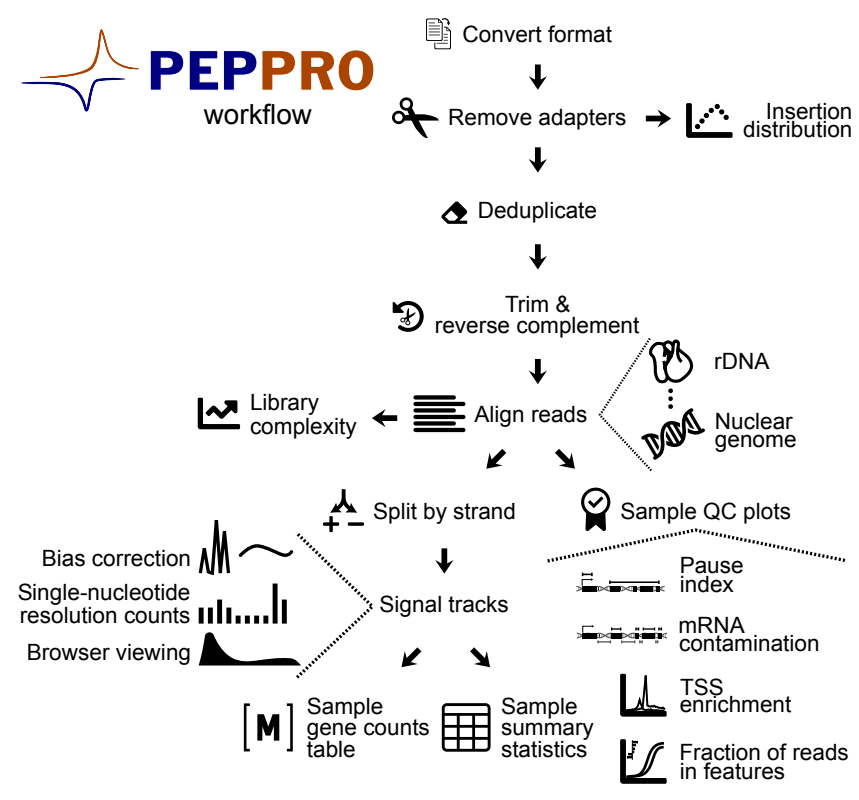

Fig. 1: PEPPRO provides a comprehensive set of steps to process genomic run on data. PEPPRO performs basic data processing starting from raw sequencing reads and produces a variety of quality control plots and processed output files that facilitate more detailed downstream analysis.

\section{Results}

\section{Pipeline overview and data description}

PEPPRO starts from raw, unaligned reads, and produces a variety of output formats, plots, and quality control metrics. Briefly, pre-alignment steps include removing adapters, deduplicating, trimming, and reverse complementation (Fig. 1). PEPPRO then uses a serial alignment strategy to siphon off unwanted reads from rDNA, mtDNA, and any other user-provided decoy sequences. It aligns reads and produces signal intensity tracks as both single-nucleotide counts files and smoothed normalized profiles for visualization. PEPPRO also provides a variety of plots and statistics to assess several aspects of library quality, such as complexity, adapter abundance, RNA integrity and purity, and run-on efficiency (See Methods for complete details).
To evaluate PEPPRO on different library types, we assembled a test set of run-on libraries with diverse characteristics (Fig. 2A). Our test set includes 7 previously published libraries: 2 ChRO-seq, 2 GRO-seq, and 3 PRO-seq ${ }^{5,12-14}$. We ran each of these samples through PEPPRO as a test case and visualized the data in a genome browser (Fig. 2B). To demonstrate PEPPRO's setup for differential expression analysis, we also generated paired-end PRO-seq libraries from H9 cell culture samples either naive or treated with romidepsin, a histone deacetylase inhibitor (HDACi). This test set therefore provides a range of qualities, protocols, and issues, providing a good test case for demonstrating the novel quality control features of PEPPRO and how to distinguish high-quality samples.

To demonstrate how PEPPRO responds to mRNA contamination, we also generated a set of 11 samples built from a single PRO-seq library (GSM1480327) that we spiked with increasing amounts of RNA-seq data (GSM765405) (Fig. S1). We ran PEPPRO on our public test set, our differential expression test set, and our spike-in set. Results of PEPPRO can be explored in the PEPPRO HTML-based web report, which displays all of the output statistics and QC plots (see PEPPRO documentation). Here, we describe each plot and statistic produced by PEPPRO.

\section{Adapter ratio}

A common source of unwanted reads in PRO/GRO/ChROseq libraries results from adapter-adapter ligation. These methods require two independent ligation steps to fuse distinct RNA adapters to each end of the nascent RNA molecule. The second ligation can lead to adapteradapter ligation products that are amplified by PCR. The frequency of adapter-adapter ligation can be reduced by molecular techniques (see Methods), but these are not always possible and many experiments retain adapters in high molar excess, leading to substantial adapter-adapter sequences.

\begin{tabular}{rrll} 
A \# & Sample name & Description & Source \\
\hline 1 & K562 PRO-seq & high quality PRO-seq data & GSM1480327 \\
2 & K562 GRO-seq & high quality GRO-seq data & GSM1480325 \\
3 & HelaS3 GRO-seq & low quality across multiple metrics & GSM1558746 \\
4 & Jurkat ChRO-seq 1 & high quality ChRO-seq data & GSM3309956 \\
5 & Jurkat ChRO-seq 2 & relatively low complexity & GSM3309957 \\
6 & HEK PRO-seq & intact RNA & GSM3618147 \\
7 & HEK ARF PRO-seq & degraded RNA & GSM3618143 \\
8 & H9 PRO-seq 1 & GSM4214080 \\
9 & H9 PRO-seq 2 \\
10 & H9 PRO-seq 3 & Differential expression analysis & GSM4214081 \\
11 & H9 treated PRO-seq 1 & GSM4214082 \\
12 & H9 treated PRO-seq 2 & & GServed $\triangle$ pause index following treatment \\
13 & H9 treated PRO-seq 3 & & GSM4214083 \\
& & GSM4214084
\end{tabular}

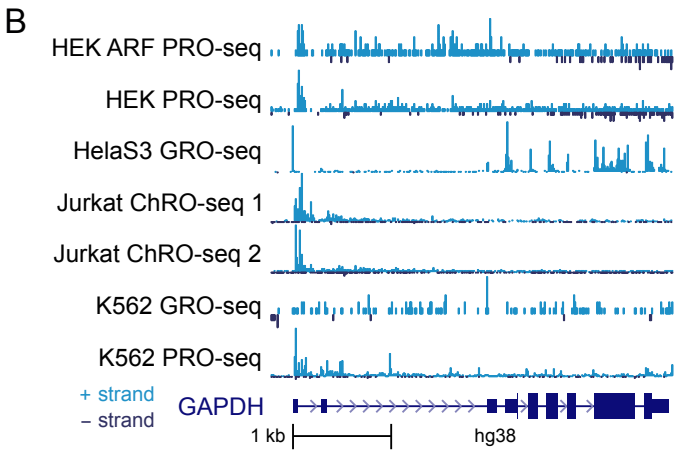

Fig. 2: PEPPRO test set data table and signal tracks. A) Table showing the attributes of samples collected for our test set. Complete metadata is available from the PEPPRO website. B) Read count normalized sample signal tracks from previously published test sets are visualized within a browser. 
A

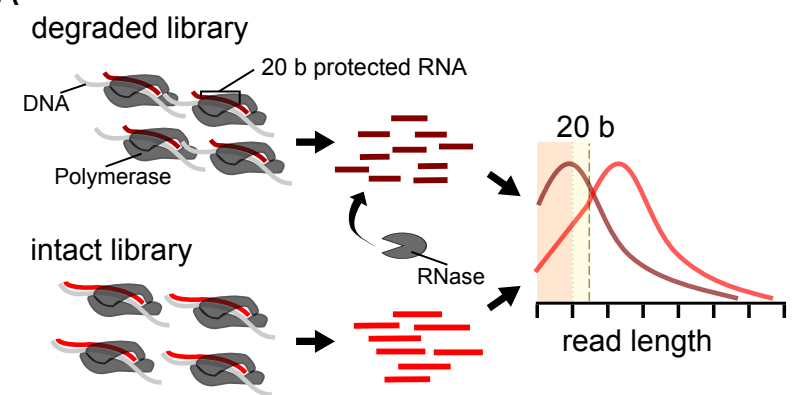

B

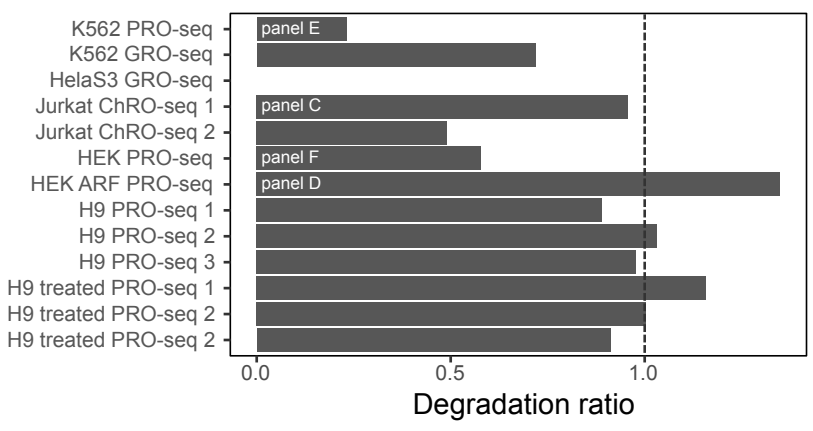

C

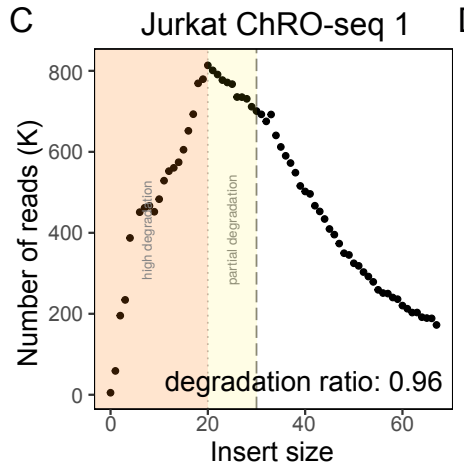

E

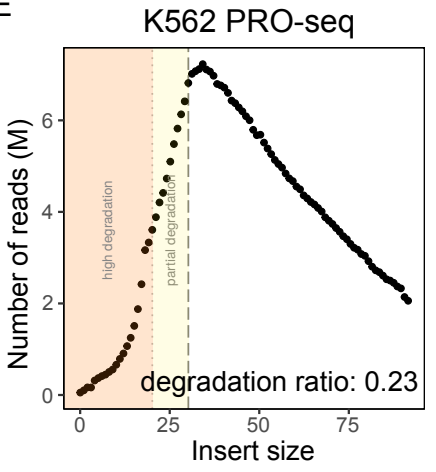

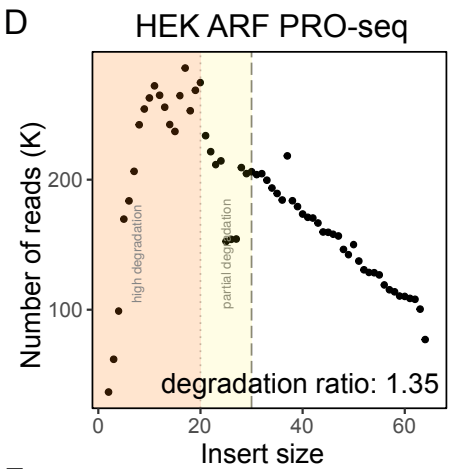

$\mathrm{F}$

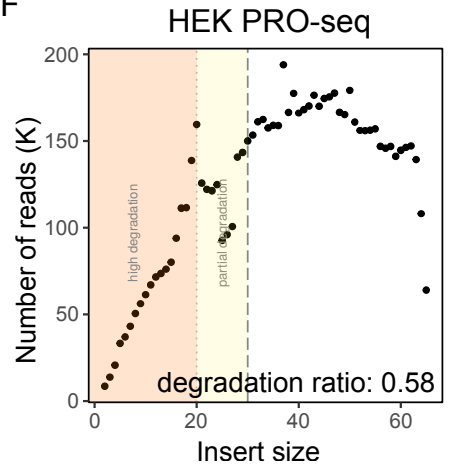

Fig. 3: RNA integrity is assessed with degradation ratios and insert sizes. A) Schematic illustrating intact versus degraded libraries. B) Degradation ratio for test samples (HelaS3 GRO sample could not be calculated; Values less than dashed line (1.0) are considered high quality). C-F) Insert size distributions for: C, a degraded single-end library; $D$, a degraded paired-end library; E, a non-degraded single-end library; and F, a non-degraded paired-end library (orange shading represents highly degraded reads; yellow shading represents partially degraded reads).

PEPPRO counts and reports the fraction of reads that contain adapter-adapter ligation products then removes adapter sequences and adapter-adapter ligation sequences before downstream alignment. In our test, all samples had fewer than 50\% adapter-adapter ligation reads (Fig. S2). Higher rates do not necessarily reflect lower quality samples, but rather indicate a suboptimal ratio of adapters during the library preparation or exclusion of the gel extraction size selection step. Excess adapters indicate that future sequencing will be less informative, leading to increased depth requirements, and therefore inform on whether to sequence a library deeper, tweak the adapter ratio in future samples, or include a size selection step. In our hands, we aim for adapter-adapter ligation abundance between 20-50\% with no size selection step, or less than $5 \%$ if the final library is polyacrylamide gel electrophoresis (PAGE) purified. Libraries with no adapter-adapter ligation indicate that size selection was too stringent, and may actively select against short RNA insertions from specific classes of nascent RNA, such as RNAs from promoter-proximal paused polymerases ${ }^{15}$.

\section{RNA integrity}

A common indicator of RNA sample quality is the level of RNA integrity. RNA integrity can be assessed by plotting the distribution of RNA insert sizes, which will be smaller when RNA is degraded. For a highly degraded library, we expect insert sizes below 20 nucleotides, which corresponds to the length of RNA between the RNA polymerase exit channel and $3^{\prime}$ RNA end. These nucleotides are sterically protected from degradation ${ }^{16}$, so high frequency of insert sizes below 20 indicates that degradation occurred after the run-on step ${ }^{5}$ (Fig. 3A).

PEPPRO uses a novel method to calculate the insert size distribution that applies to both single- and paired-end data (see Methods). PEPPRO reports the ratio of insert sizes from 10-20 nucleotides versus 30-40 nucleotides, which measures RNA integrity because more degraded libraries have higher frequency of reads of length 1020, whereas less degraded libraries have more reads of length 30-40. Using our test set, we found that PROseq libraries with a ratio $<1$ should be considered high quality (Fig. 3B). A single-end ChRO-seq library that was intentionally degraded with RNase prior to the run on step ${ }^{5}$ has a degradation ratio near 1 with a insertion distribution plot showing a peak at 20 nucleotides (Fig. 3C). A poor quality paired-end PRO-seq library contains many RNA species falling within the 10-20 range (Fig. 3D). High-quality libraries show plots that peak outside of the sub-20-nucleotide degradation zone (Fig. 3E, F). 
A

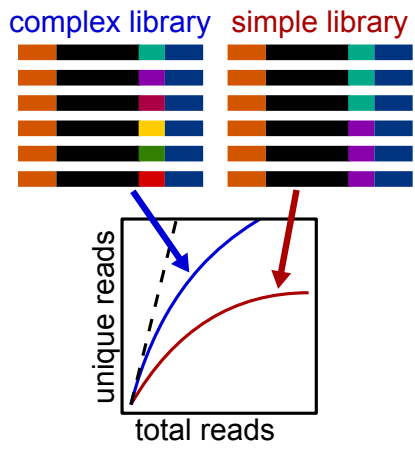

$\mathrm{B}$

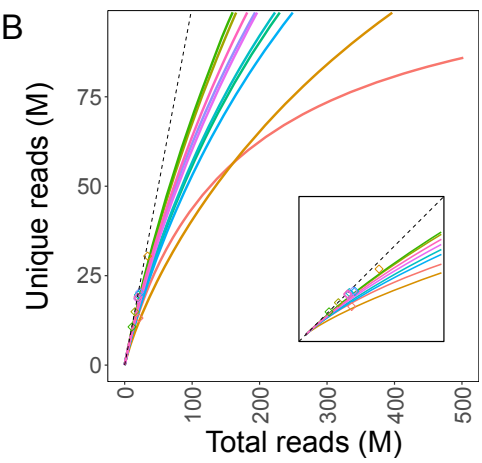

C

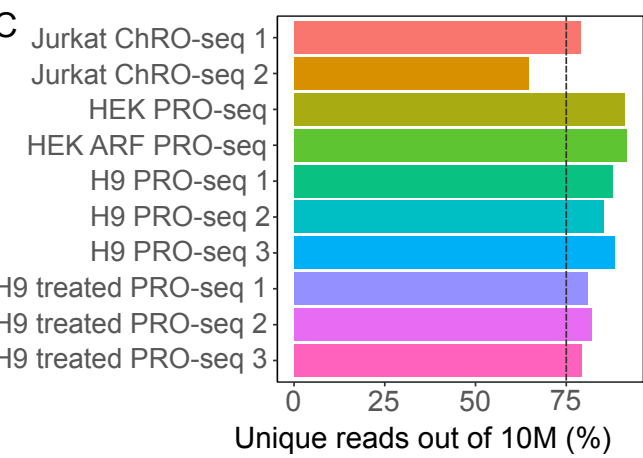

Fig. 4: Library complexity is measured with unique read frequency distributions and projections. A) Schematic demonstrating $P C R$ duplication and library complexity (dashed line represents completely unique library). B) Library complexity traces plot the read count versus externally calculated deduplicated read counts. Deduplication is a prerequisite, so these plots may only be produced for samples with UMIs. Inset zooms to region from 0 to double the maximum number of unique reads. C) The position of curves in panel $B$ at a sequencing depth of 10 million reads (dashed line represents minimum recommended percentage of unique reads).

\section{Library complexity}

Library complexity measures the uniqueness of molecules in a sequencing library (Fig. 4A). For conventional RNA-seq, shearing is random, so pairedend reads with the same start and end coordinates may be assumed to be PCR duplicates. In contrast, in PRO-seq, transcription start sites account for many of the $5^{\prime}$ RNA ends, and promoter proximal pause sites can focus the $3^{\prime}$ end of the RNA ${ }^{4}$, so independent insertions with the same end points are not necessarily PCR duplicates. As a result, unfortunately, it is not possible to calculate complexity generally due to the biology of nascent RNA-seq.

Recent PRO-seq protocols resolve this by incorporating a unique molecular identifier (UMI) into the $3^{\prime}$ adapter, which PEPPRO uses to distinguish between PCR duplicates and independent RNA molecules with identical ends. For data that includes UMIs, PEPPRO accommodates multiple software packages for read deduplication, including seqkit ${ }^{17}$ and fqdedup ${ }^{18}$. PEPPRO calculates library complexity at the current depth, reporting the percentage of PCR duplicates. In our test samples, we found that libraries with at least $75 \%$ of reads unique at a sequencing depth of 10 million can be considered high quality (Fig. 4C). PEPPRO also invokes preseq ${ }^{19}$ to project the unique fraction of the library if sequenced at higher depth (Fig. 4B). These metrics provide a direct measure of library complexity and allow the user to determine value of additional sequencing. However, because nascent RNA reads cannot be effectively deduplicated using the standard approach applied to traditional RNA-seq, complexity metrics are only calculated for samples with UMIs.

\section{Nascent RNA purity}

One challenge specific to nascent RNA sequencing is ensuring that the library targets nascent RNA specifically, which requires eliminating the more abundant processed rRNA, tRNA, and mRNA transcripts. Early run-on protocols included 3 successive affinity purifications, resulting in 10,000-fold enrichment over mRNA and over $98 \%$ purity of nascent $\mathrm{RNA}^{3,4}$. Newer run-on protocols recommend fewer affinity purifications ${ }^{14}$. Therefore, assessing the efficiency of nascent enrichment is a useful quality control output.

To estimate the nascent purity of RNA, PEPPRO provides two results: rDNA alignment rate and an mRNA contamination metric. First, since rRNA represents the vast majority of stable RNA species in a cell, overrepresentation of rDNA reads indicates poor nascent RNA enrichment. We find that high-quality nascent RNA libraries typically have less than 20\% rRNA alignment (Fig. S3). In contrast, between $70 \%$ and $80 \%$ of mature RNA in a cell is rRNA. Therefore, the ratio of rDNA aligned reads compared to the all other reads reflects mature RNA contamination, with the caveat that the fraction of nascent rRNA transcription is likely to be distinct among cell lines. A second measure of nascent purity is to evaluate messenger RNA abundance.

PEPPRO assesses this by calculating the exon to intron read density ratio (Fig. 5A). A nascent RNA sequencing library without polymerase pausing would have a ratio of exon density to intron density of $\approx 1$. Because promoter-proximal pausing inflates this ratio, PEPPRO excludes the first exon from this calculation. In our test samples, the median exon-intron ratio is between 1.0 and 1.8 for high quality libraries (Fig. 5B). Our in silico spike-in of conventional RNA-seq increases this ratio proportionally to the level of mRNA contamination (Fig. 5B). This ratio varies substantially among genes and PEPPRO produces histograms to compare in more detail among samples (Fig. 5C-F). By comparing these values to the spike-in experiment, we can estimate the level of mRNA contamination of a library (Fig. 5E, F). 
bioRxiv preprint doi: https://doi.org/10.1101/2020.02.27.956110; this version posted July 15, 2020. The copyright holder for this preprint (which was not certified by peer review) is the author/funder, who has granted bioRxiv a license to display the preprint in perpetuity. It is made available under aCC-BY 4.0 International license.

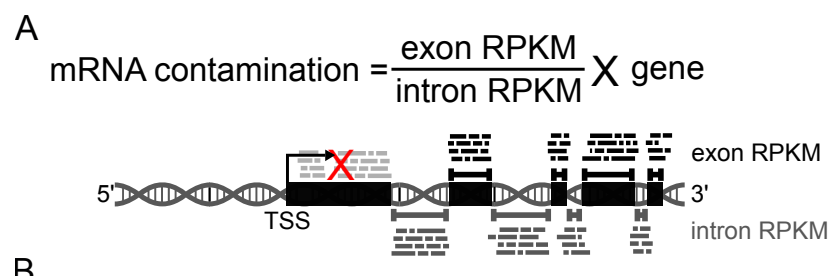

B

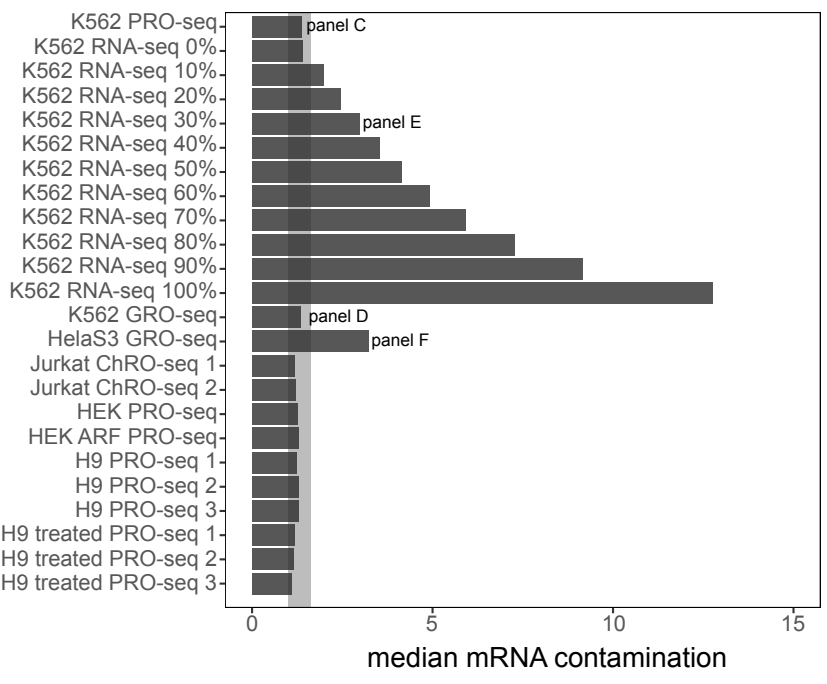

C
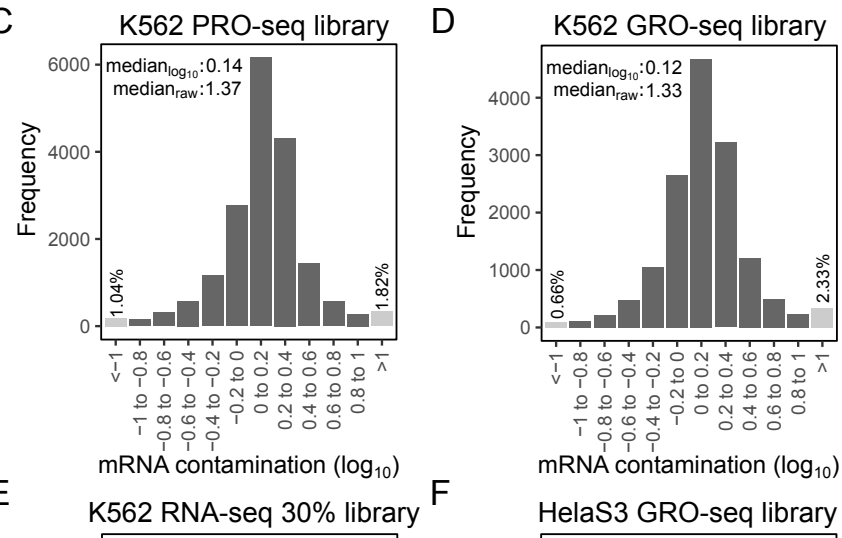

$F$

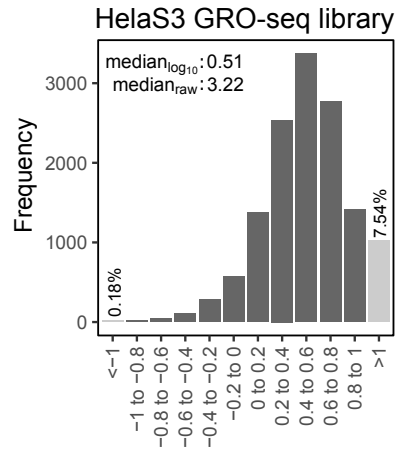

Fig. 5: Nascent RNA purity is assessed with the exon-intron ratio. A) Schematic demonstrating mRNA contamination calculation. B) Median mRNA contamination metric for test set samples (Shaded region represents recommended range (1-1.8)). C) Histogram showing the distribution of mRNA contamination score across genes in the K562 PRO-seq sample. D) As in panel C for a GRO-seq library. E) mRNA contamination distribution for K562 PRO-seq spiked with 30\% K562 RNA-seq. F) mRNA contamination distribution for HelaS3 GRO-seq is comparable to the 30\% RNA-seq spike-in sample.

A

$$
\text { Pause index }=\frac{\text { pause window RPKM }}{\text { gene body RPKM }}
$$

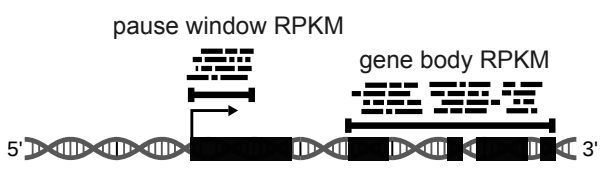

B

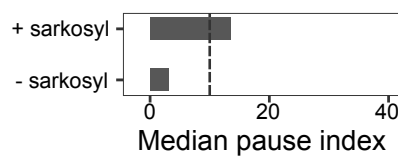

C

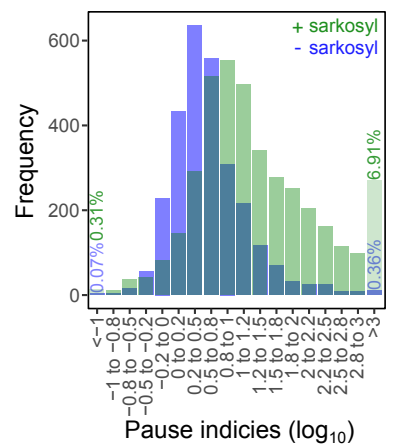

D

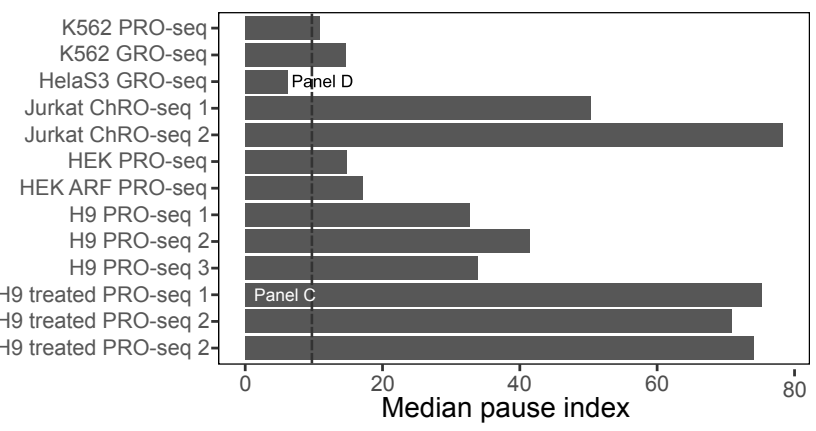

E

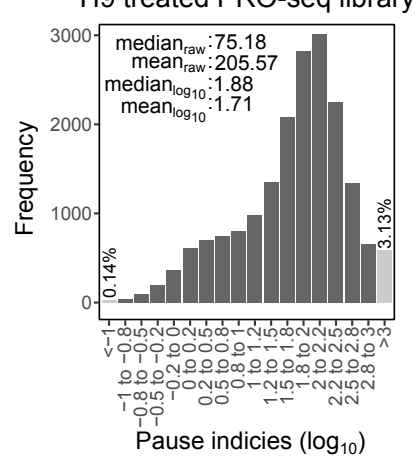

$\mathrm{F}$

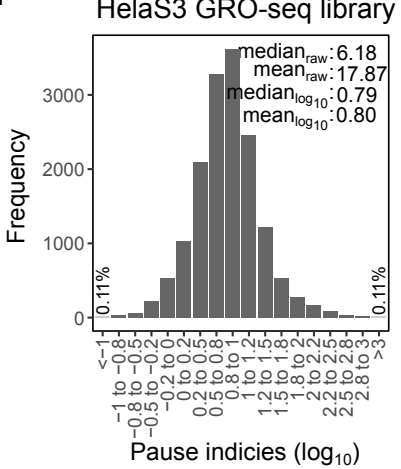

Fig. 6: Run-on efficiency is measured with pause indices. A) Schematic demonstrating pause index calculation. B) Pause index values for Drosophila melanogaster GRO-seq libraries with (GSM577247) or without sarkosyl (GSM577248). C) The histogram of pause index values is shifted to the right upon addition of sarkosyl in GRO-seq libraries. D) Pause index values for test set samples (Values above the dashed line are recommended). E) High pause index identified in H9 treated PRO-seq. F) Low pause index from HelaS3 GRO-seq. 


\section{Run-on efficiency}

Another quality metric for run-on experiments is run-on efficiency. Typically, gene-body polymerases extend efficiently during the nuclear run-on step, but promoterproximal paused polymerases require either high salt or detergent to do $\mathrm{so}^{20,21}$. Because these treatments may vary, PEPPRO employs two methods to assess run-on efficiency: pause index and TSS enrichment. First, PEPPRO calculates the pause index, which is defined as the ratio of the density of reads in the pausing region versus the density in the corresponding gene body (Fig. 6A; see Methods). PEPPRO plots the frequency distribution of the pause index across genes. A greater pause index indicates a more efficient run-on, as a higher value indicates that paused polymerases efficiently incorporate the modified NTPs. As test of this metric, we analyzed GROseq data that was generated in the presence and absence of the anionic detergent Sarkysol ${ }^{21}$. Paused polymerases necessitate detergent to run on and incorporate NTPs efficiently, thus the pause index drops substantially in the absence of Sarkysol (Fig. 6B\&C). We found in our test samples that an efficient run-on process has a median pause index greater than 10 (Fig. 6D). For more detail, PEPPRO produces frequency distribution plots that show an exponential distribution among genes for an efficient library (or a normal distribution on a log scale, Fig. 6E) and a shifted distribution for an inefficient run-on (Fig. $6 \mathrm{~F})$.

As a second assessment of run-on efficiency, PEPPRO aggregates sequencing reads at TSSs to plot and calculate a TSS enrichment score. PEPPRO plots aggregated reads 2000 bases upstream and downstream of a reference set of TSSs. The normalized TSS enrichment score is calculated by taking the average base coverage in a $100 \mathrm{bp}$ window around the peak divided by the average coverage in the first 200 bases. Efficient TSS plots show a characteristic PRO-seq pattern with an upstream peak for divergently transcribing polymerases and a prominent peak representing canonical paused polymerases (Fig. S4). PEPPRO also summarizes these values across samples.

\section{Read feature distributions}

PEPPRO also produces plots to visualize the fraction of reads in features, or FRiF. The cumulative FRiF (cFRiF) plot provides an information-dense look into the genomic distribution of reads relative to genomic features. This analysis is a generalization of the more common fraction of reads in peaks (FRiP) plots produced for other data types ${ }^{22}$ with two key differences: First, it shows how the reads are distributed among different features, not just peaks; and second, it uses a cumulative distribution to visualize how quickly the final read count is accumulated in features of a given type. To calculate the FRiF, PEPPRO overlaps each read with a feature set of genomic annotations, including: enhancers,
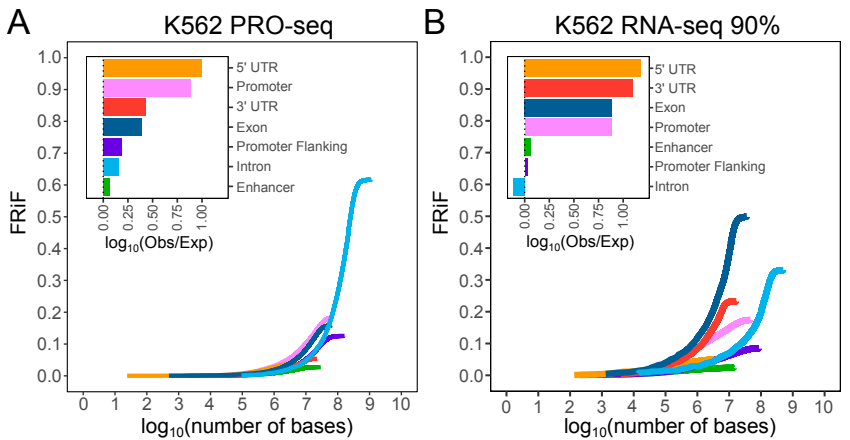

Fig. 7: Fraction of reads in genomic features. A) K562 PROseq represents a "good" cumulative fraction of reads in features (cFRiF) and fraction of reads in features (FRiF) plot. B ) K562 PRO-seq with 90\% K562 RNA-seq spike-in represents a "bad" FRiF/PRiF.

promoters, promoter flanking regions, $5^{\prime}$ UTR, $3^{\prime}$ UTR, exons, and introns (Fig. 7). The individual feature elements are then sorted by read count, and for each feature, we traverse the sorted list and calculate the cumulative sum of reads found in that feature divided by the total number of aligned reads. We plot the read fraction against the $\log _{10}$ transformed cumulative size of all loci for each feature. This allows the identification of features that are enriched for reads with fewer total features and total genomic space. Additionally, PEPPRO calculates the non-cumulative FRiF by taking the $\log _{10}$ of the number of observed bases covered in each feature over the number of expected bases in each feature to identify enriched genomic features (Fig. 7).

In our test samples, high-quality libraries have a characteristic pattern with slow accumulation but high total of reads in introns, and fast accumulation but lower total of reads in promoter elements. ChRO-seq libraries have an increased promoter emphasis and higher mRNA contamination indicated by an increase in reads in promoters and exons at the cost of reads in introns and promoter flanking regions (Fig. S5). Additionally, the RNA-seq spike-in samples demonstrate the increasing prevalence of exonic reads and $3^{\prime}$ UTR at the cost of intronic sequences (Fig. S6). These plots are therefore a useful general-purpose quality control tool that reveal substantial information about a sample in a concise visualization.

\section{Differential expression}

The focus of PEPPRO is in the pre-processing relevant for any type of biological project. The output of PEPPRO sets the stage for downstream analysis specific to a particular biological question. Perhaps the most common downstream application of nascent transcription data is differential expression analysis. PEPPRO allows the user to easily run a differential comparison using dedicated software like the DESeq bioconductor package ${ }^{23}$. To demonstrate this, we included PRO-seq libraries from 

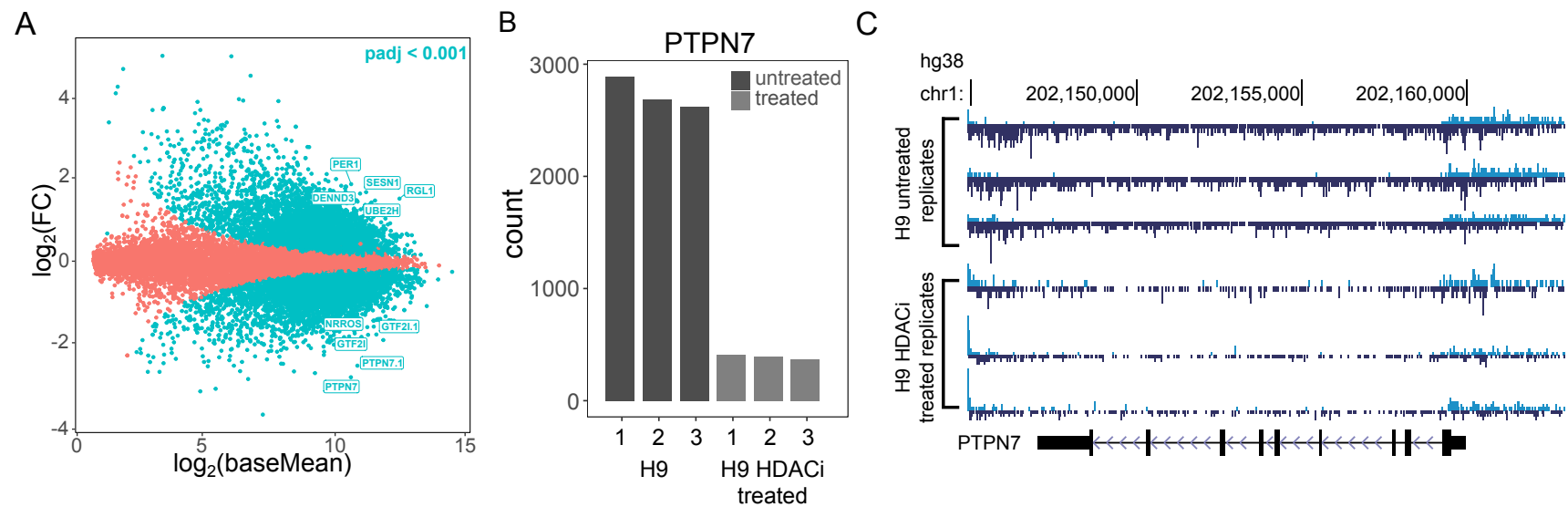

Fig. 8: Differential analysis with the PEPPRO counts matrix. A) MA plot between H9 DMSO versus H9 200 M romidepsin treated PRO-seq libaries (dots = genes; top 10 most significant genes labeled; $n=3 /$ treatment). B) Most significantly differential gene count differences. C) Read count normalized signal tracks from the differential analysis.

\begin{aligned} Metric & Recommended value \\ \hline Degradation ratio & $<1 \\$ rDNA alignment rate & $<20 \% \\$ Pause index & $>10 \\$ mRNA contamination & $1-1.8 \\ \%$ uniformative adapter reads (PAGE) & $<5 \% \\ \%$ uniformative adapter reads (w/o PAGE) & $20-50 \% \\ \%$ unique at 10M reads & $>75 \%\end{aligned}$

Fig. 9: Recommendation table. Based on our experience processing both high- and low-quality nascent RNA libraries, these are the recommended values for high-quality $P R O$-seq libraries.

H9 human cutaneous T-cell lymphoma cell lines treated with either DMSO $(n=3)$ or an HDAC inhibitor $(n=3)$.

To facilitate differential expression analysis, PEPPRO produces a project-level counts table that may be loaded in R using pepr, and, in a few lines of code, converted quickly into DEseq data sets ready for downstream DESeq analyses (See Supplemental text). Using this approach, we ran a differential expression analysis comparing romidepsin-treated against untreated samples (Fig. 8A). We identified many genes with significantly different read coverage. As an example, the PTPN7 gene showed clear differences in counts (Fig. 8B), which we can further visualize using the browser track outputs generated by PEPPRO (Fig. 8C). This analysis demonstrates how simple it is to ask a downstream biological question starting from the output produced by PEPPRO.

\section{Metric robustness}

To evaluate the robustness of our metrics across sequencing depth and library complexity, we ran PEPPRO on subsampled single-end and paired-end with UMI PRO-seq libraries (Fig. S7, Fig. S8). Our metrics remained constant across sequencing depth from as few as $10 \mathrm{M}$ reads to well over $100 \mathrm{M}$ (Fig. S9, Fig. S10). We also generated synthetic low complexity paired-end with UMI PRO-seq libraries and our metrics remain robust to reductions in library complexity (Fig. S11).

Because our metrics are based on specific source annotation files, we also investigated the effect of alternative annotation source files. To illustrate, we recalculated exon:intron density ratios and pause indicies using UCSC RefSeq, Ensembl, and GENCODE gene set annotation files. While specific values per sample may have minor changes, as would be expected, the relationship between samples remains constant (Fig. S12).

\section{Conclusions}

PEPPRO is an efficient, user-friendly PRO/GRO/ChROseq pipeline that produces novel, integral quality control plots and signal tracks that provide a comprehensive starting point for further downstream analysis. The included quality control metrics inform on library complexity, RNA integrity, nascent RNA purity, and run-on efficiency with theoretical and empirical recommended values (Fig. 9). PEPPRO is uniquely flexible, allowing pipeline users to serially align to multiple genomes, to select from multiple bioinformatic tools, and providing a convenient configurable interface so a user can adjust parameters for individual pipeline tasks. Furthermore, PEPPRO reads projects in PEP format, a standardized, well-described project definition format, providing an interface with Python and R APIs to simplify downstream analysis.

PEPPRO is easily deployable on any compute infrastructure, including a laptop, a compute cluster, or the cloud. It is thereby inherently expandable from single to multi-sample analyses with both group level and individual sample level quality control reporting. By design, PEPPRO enables simple restarts at any step in the process should the pipeline be interrupted. At multiple steps within the pipeline, different software options exist creating a swappable pipeline flow path with individual steps adaptable to future changes in 
the field. PEPPRO is a rapid, flexible, and portable $\mathrm{PRO} / \mathrm{GRO} / \mathrm{ChRO}$-seq project analysis pipeline providing a standardized foundation for more advanced inquiries.

\section{Availability}

Documentation on the Portable Encapsulated Project (PEP) standard may be found at pepkit.github.io.

Refgenie documentation and pre-built reference genomes are available at refgenie.databio.org.

The HTML report for the test samples is located at http://big.databio.org/papers/peppro/PEPPRO_ summary.html.

\section{Methods}

\section{Pipeline implementation}

The PEPPRO pipeline is a python script (peppro.py) runnable from the command-line. PEPPRO provides restartability, file integrity protection, logging, monitoring, and other features. Individual pipeline settings can be configured using a pipeline configuration file (peppro.yaml), which enables a user to specify absolute or relative paths to installed software and parameterize alignment and filtering software tools. Required software includes several Python packages (cutadapt ${ }^{24}$, looper, numpy ${ }^{25}$, pandas ${ }^{26}$, pararead, pypiper, and refgenie ${ }^{27}$ ) and $\mathrm{R}$ packages (installed via the included PEPPROr $\mathrm{R}$ package) in addition to some common bioinformatics tools including bedtools ${ }^{28}$, bigWigCat ${ }^{29}$, bowtie $2^{30}$, fastq-pair ${ }^{31}$, flash ${ }^{32}$, picard, preseq ${ }^{19}$, seqkit $^{17}$, samtools ${ }^{33}$, seqtk, and wigToBigWig ${ }^{29}$. This configuration file will work out-of-the-box for research environments that include required software in the shell PATH, but may be configured to fit any computing environment and is adaptable to project-specific parameterization needs.

\section{Refgenie reference assembly resources}

Several PEPPRO steps require generic reference genome assembly files, such as sequence indexes and annotation files. For example, alignment with bowtie2 requires bowtie 2 indexes, and feature annotation to calculate fraction of reads in features requires a feature annotation. To simplify and standardize these assembly resources, PEPPRO uses refgenie. Refgenie is a reference genome assembly asset manager that streamlines downloading, building, and using data files related to reference genomes ${ }^{27}$. Refgenie includes recipes for building genome indexes and genome assets as well as downloads of pre-indexed genomes and assets for common assemblies. Refgenie enables easy generation of new standard reference genomes as needed. For a complete analysis, PEPPRO requires a number of refgenie managed assets. Those assets as defined by refgenie are: fasta, bowtie2_index, ensembl_gtf, ensembl_rb, refgene_anno, and feat_annotation. If building these assets manually, they separately require a genome fasta file, a gene set annotation file from RefGene, an Ensembl gene set annotation file in GTF format, and an Ensembl regulatory build annotation file. Finally, using PEPPRO with seqOutBias requires the additional refgenie tallymer_index asset of the same read length as the data.

\section{Adapter-adapter ligation product abundance}

Adapter-adapter ligation products show up in run-on libraries because there are two independent ligation steps. Sequencing these products is uninformative, and so there are several molecular approaches used to reduce their abundance in a sequencing library. All protocols include an inverted dT on the $3^{\prime}$ end of the $3^{\prime}$ adapter, and also do not phosphorylate the $5^{\prime}$ end of the $5^{\prime}$ adapter. Many protocols include a size-selection gel extraction step to purify the library from a prominent adapter-adapter ligation species.

PEPPRO calculates adapter-adapter ligation products directly from cutadapt output, and the default -m value for this step is the length of the UMI plus two nucleotides. Therefore, if RNA insertions fewer than three nucleotides in length are present in the library, these are treated as adapter-adapter ligation products.

\section{RNA insert size distribution and degradation}

For both single and paired end data, the RNA insert size distribution is calculated prior to alignment. For single end data, the calculation is derived only from sequences that contain adapter sequence, which is output directly from cutadapt ${ }^{24}$. PEPPRO plots the inverse cutadapt report fragment lengths against the cutadapt fragment counts. If there is a known UMI, based on user input, that length is subtracted from reported cutadapt fragment lengths. As a consequence of this distribution, we can establish a measure of library integrity by evaluating the sum of fragments between 10-20 bases versus the sum of fragments between $30-40$ bases in length. The higher this degradation ratio, the more degraded the library.

Paired end sequencing files often have shorter reads because a standard 75 base sequencing cartridge can be used for two paired end reads that are each 38 nucleotides in length. Therefore, many fewer of the reads derived from either end of the molecule extend into the adapter sequence. To address this issue, we incorporate a step that fuses overlapping reads using $\mathrm{flash}^{32}$. Therefore, if two paired end reads contain overlapping sequence, the reads are combined and the insert size is calculated directly from the fused reads and output directly from flash. This distribution is plotted identically to the single end reads and degradation is calculated in the same manner. This degradation ratio metric is uniform between single-end or paired-end 
libraries and is reported prior to any alignment steps, minimizing influences from extensive file processing or alignment eccentricities.

\section{Excluding size selection skews metrics}

Recent PRO-seq protocols, including the H9 libraries we generated, exclude the PAGE size selection step that removes adapter-adapter ligation products ${ }^{14}$. Size selection can potentially bias against small RNA insertions. The previous two metrics: adapter-adapter abundance and degradation ratio are naturally skewed toward the undesirable range if libraries are constructed without size selection. Adapter abundance is skewed because the sole purpose of size selection is to remove the adapter species, but these uninformative reads are of minimal concern and can be overcome by increasing sequencing depth. Degradation ratio is skewed higher because the size selection is not perfect and insert sizes in the range of 10-20 are preferentially selected against relative to those in the 30-40 range. Therefore, while we provide recommendations for optimal degradation ratios, this metric is not necessarily comparable between library preparation protocols and a higher ratio is expected for protocols that exclude size selection.

\section{Removing UMI and reverse complementation}

In a typical sequencing library, low library complexity is indicated by high levels of PCR duplicates. Conventional methods remove independent paired-end reads that map to the same genomic positions. This method works reasonably well for molecular genomics data sets with random nucleic acid cleavage. However, in PRO-seq, transcription start sites account for many of the $5^{\prime}$ RNA ends and polymerases pause downstream in a focused region ${ }^{4}$. Consequently, independent insertions with the same end points are common, especially in the promoter-proximal region. To solve this, PRO-seq protocols incorporate a unique molecular identifier (UMI) into the $3^{\prime}$ adapter to distinguish between PCR duplicates and independent insertions with shared ends. PEPPRO removes PCR duplicates only if UMIs are provided.

Following the removal of PCR duplicates, the UMI is trimmed. For run-on experiments where the sequencing primer sequences the $3^{\prime}$ end of the original RNA molecule, reverse complementation is performed. As only the first read contains a UMI in paired-end experiments, the second reads skip UMI trimming. Both steps are performed using either seqtk (https://github.com/lh3/seqtk) or fastx (https://github.com/agordon/fastx_toolkit),

depending on user preference. Because reads are processed uniquely for first and second reads in a paired-end experiment, reads must be re-paired prior to alignment. PEPPRO uses the optimized implementation fastq-pair ${ }^{31}$ to re-pair desynchronized read files.

\section{Serial alignments}

Following re-pairing, or starting from processed singleend reads, PEPPRO performs a series of preliminary, serial alignments (prealignments) before aligning to the primary reference using bowtie $2^{30}$. As a significant portion of nascent transcription includes rDNA, PEPPRO defaults to initially aligning all reads to the human rDNA sequence. Not only does this remove rDNA reads from downstream analysis, it improves computational efficiency by aligning the largest read pool to a small genome and reduces that read pool for subsequent steps. The user can specify any number of additional genomes to align to prior to primary alignment, which may be used for species contamination, dual-species experiments, repeat model alignments, decoy contamination, or spike-in controls. For serial alignments, bowtie 2 is run with the following parameters $-k 1$ -D $20-R \quad 3-N \quad 1-L 20-i \mathrm{~S}, 1,0.50$, where we are interested primarily in quickly identifying and removing any reads that have a valid alignment to the serial alignment genome (-k 1 parameter). These settings are easily adjusted in the pipeline configuration file (peppro.yaml).

Subsequent to these serial alignments, remaining reads are aligned to the primary genome. Primary genome alignment uses the bowtie2 --very-sensitive option by default and sets the maximum paired-end fragment length to 2000 . The goal with primary alignment is to identify the best valid alignment for reads, sacrificing speed for accuracy. Following primary alignment, low-quality reads are removed using samtools view -q 10. As with the initial prealignments, these parameters can be customized by the user in the pipeline configuration file (peppro.yaml). Alignment statistics (number of aligned reads and alignment rate) for all serial alignments and primary alignments are reported. For the primary alignment, PEPPRO also reports the number of mapped reads, the number removed for quality control, the total efficiency of alignment (aligned reads out of total raw reads), and the read depth. Prior to further downstream analysis, paired-end reads are split into separate read alignment files and only the first read is retained for downstream processing. For both pairedend and single-end experiments, this aligned read file is split by strand with both plus and minus strand aligned files further processed.

\section{Processed signal tracks}

Following read processing, alignment, strand separation, and quality control reporting, aligned reads are efficiently converted into strand-specific bigWig files by default. For PRO-seq and similar protocols, reads are reported from the $3^{\prime}$ end and may optionally be scaled by total reads. PEPPRO may alternatively use seqOutBias ${ }^{18}$ to correct enzymatic sequence bias. Bias is corrected by taking the ratio of genome-wide 
observed read counts to the expected sequence based counts for each k-mer ${ }^{18}$. K-mer counts take into account mappability at a given read length using Genome Tools' Tallymer program ${ }^{34}$. Correcting for enzymatic bias can be important as bias from T4 RNA Ligase used in PRO-seq protocols can yield erroneous conclusions ${ }^{18}$. As such, we recommend using seqDutBias for bias correction when analyzing a typical PRO-seq library. Bias correction is especially important when plotting composite profiles over sequence features. Strand specific bigWigs may be visually analyzed using genomic visualization tools and provide a unified starting point for downstream analyses. For example, output bigWig files can be directly loaded into AREG to identify regulatory elements defined by bidirectional transcription ${ }^{1}$.

\section{Exon-intron ratio plots}

PEPPRO provides an mRNA contamination histogram for quick visual quality control, and a BED format file containing gene by gene exon:intron ratios for detailed analysis. To calculate this metric, PEPPRO utilizes annotation files derived from UCSC RefSeq gene files. Because promoter-proximal pausing inflates these ratios, PEPPRO excludes the first exon from the calculation. Otherwise, the reads per kilobase per million mapped reads (RPKM) is calculated for all exonic and intronic sequences on a gene by gene basis. Then, the ratio of exon RPKM to intron RPKM is determined for every gene. The overall measure, the mRNA contamination metric, is the median of all genic exon:intron density ratios.

\section{Pause index}

Pause indices are calculated as the ratio of read density in the promoter proximal region versus read density in the gene body. To calculate these values, PEPPRO utilizes annotation files derived from Ensembl gene set files. Pause indices can vary widely depending on the defined pause window and how a pause window is determined (i.e. relative to a TSS or the most dense window proximal to a TSS). PEPPRO defines the density within the pause region as the single, most dense window $+20-120 \mathrm{bp}$ taken from all annotated TSS isoforms per gene. This is necessary as some genes contain multiple exon 1 annotations and because this region is where most polymerase pausing occurs, PEPPRO identifies the predominant exon 1, based on density, and calculates the pause index using this window density. This means that for genes with multiple TSSs, we define the pause window as the region $+20-120$ bases from each identified TSS per gene. We determine the read density at every annotated pause window per gene, and identify the predominant, singular pause window as the pause window with the greatest density. This singular pause window is used to calculate the pause index for that gene. The corresponding gene body is defined as the region beginning 500 bp downstream from the predominant TSS to the gene end. We found that lowly expressed genes represent a significant portion of genes with a low pause index. At low sequencing depth, these lowly expressed genes experience greater dropout and fluctuation in pause index calculation, skewing the metric upwards at low depth. To address this, we restrict the pause index calculation to the upper 50th percentile of genes by expression, which eliminates the variability due to depth. Finally, PEPPRO plots the distribution of pause indices for each remaining gene in a histogram and provides a BED-formatted file containing each gene's pause index for more detailed analyses.

\section{PRO-seq experiments}

H9 PRO-seq experiments were conducted as described previously ${ }^{14}$. The HDACi-treated samples were incubated with 200nM romidepsin for 60 minutes prior to harvesting. The control "untreated" samples were treated with DMSO for 60 minutes. We have included these samples as a test to demonstrate differential expression analysis using PEPPRO. They also provide additional example libraries for the metrics in general, and unexpectedly, show significant differences in pause index upon treatment.

\section{Synthetic experiments}

Synthetic sequencing depth variant libraries were constructed for single-end and paired-end PRO-seq libraries using either the K562 PRO-seq (GSM1480327) or H9 PRO-seq 2 (GSM4214081) as source libraries, respectively. For K562 PRO-seq subsamples, seqtk sample -s99 was called on the raw fastq files to generate libraries between $2-10 \%$, in 2 percent increments, and between $10-100 \%$, in 10 percent increments. For the H9 PRO-seq libraries, seqtk sample -s99 was called on the raw fastq files to produce libraries between $10-100 \%$, in 10 percent increments. Lower percentage K562 PRO-seq libraries were generated to yield libraries of total size comparable to low percentage H9 PRO-seq libraries.

RNA-seq spike-in libraries were also produced using the command seqtk sample -s99 on raw fastq files using combinations of the K562 PRO-seq library utilized prior and a corresponding K562 RNA-seq library (GSM765405). RNA-seq libraries were sampled between $10-100 \%$, in 10 percent increments, and concatenated with the sampled K562 PRO-seq libraries to generate mixed libraries composed of 0-100\% RNA-seq.

Low complexity libraries were similarly constructed. Thirty million total read libraries were generated by using seqtk sample -s99 on the H9 PRO-seq 2 library and sampling at 50, 80, 90, 92, 94, 96, 98, and 100\%. At each percentage of original H9 PRO-seq 2 library sample, the remainder represents duplicates of the 
original raw reads composing the opposite percentage, producing libraries with varying levels of duplicated reads.

\section{Funding}

This work was supported by the National Institute of General Medical Sciences grants GM128635 (MJG) and GM128636 (NCS). JPS was supported by the institutional training grant GM008136. ABD was supported by institutional training grants LM012416 and GM007267.

\section{Declarations}

All authors declare no competing interests.

\section{References}

1. Wang, Z., Chu, T., Choate, L. A. \& Danko, C. G. Identification of regulatory elements from nascent transcription using dREG. Genome Research 29, 293-303 (2019).

2. Scruggs, B. S. et al. Bidirectional transcription arises from two distinct hubs of transcription factor binding and active chromatin. Molecular Cell 58, 1101-1112 (2015).

3. Core, L. J., Waterfall, J. J. \& Lis, J. T. Nascent RNA sequencing reveals widespread pausing and divergent initiation at human promoters. Science 322, 1845-1848 (2008).

4. Kwak, H., Fuda, N. J., Core, L. J. \& Lis, J. T. Precise maps of RNA polymerase reveal how promoters direct initiation and pausing. Science 339, 950-953 (2013).

5. Chu, T. et al. Chromatin run-on and sequencing maps the transcriptional regulatory landscape of glioblastoma multiforme. Nature genetics 50, 1553-1564 (2018).

6. Chae, M., Danko, C. G. \& Kraus, W. L. GroHMM: A computational tool for identifying unannotated and cell type-specific transcription units from global run-on sequencing data. BMC Bioinformatics 16, 222 (2015).

7. Azofeifa, J. G., Allen, M. A., Lladser, M. E. \& Dowell, R. D. An annotation agnostic algorithm for detecting nascent RNA transcripts in gro-seq. IEEE/ACM transactions on computational biology and bioinformatics 14, 1070-1081 (2017).

8. Allison, K. A., Kaikkonen, M. U., Gaasterland, T. \& Glass, C. K. Vespucci: A system for building annotated databases of nascent transcripts. Nucleic acids research 42, 2433-2447 (2014).

9. Wang, P., Wang, D. \& Lu, J. Controllability analysis of a gene network for arabidopsis thaliana reveals characteristics of functional gene families. IEEE/ACM Transactions on Computational Biology and Bioinformatics 1-1 (2018). doi:10.1109/tcbb.2018.2821145
10. Anderson, W. D., Duarte, F. M., Civelek, M. \& Guertin, M. J. Defining data-driven primary transcript annotations with primaryTranscriptAnnotation in R. Bioinformatics (2020). doi:10.1093/bioinformatics/btaa011

11. Sheffield, N. C. Bulker: A multi-container environment manager. OSF Preprints (2019). doi:10.31219/osf.io/natsj

12. Core, L. J. et al. Analysis of nascent RNA identifies a unified architecture of initiation regions at mammalian promoters and enhancers. Nature genetics 46, 1311 (2014).

13. Duttke, S. H. C. et al. Human promoters are intrinsically directional. Molecular Cell 57, 674-684 (2015).

14. Sathyan, K. M. et al. An improved auxin-inducible degron system preserves native protein levels and enables rapid and specific protein depletion. Genes \& Development 33, 1441-1455 (2019).

15. Andersson, R. et al. Human gene promoters are intrinsically bidirectional. Molecular Cell 60, 346-347 (2015).

16. Choder, M. \& Aloni, Y. RNA polymerase ii allows unwinding and rewinding of the dna and thus maintains a constant length of the transcription bubble. Journal of Biological Chemistry 263, 12994-13002 (1988).

17. Shen, W., Le, S., Li, Y. \& Hu, F. SeqKit: A crossplatform and ultrafast toolkit for fasta/q file manipulation. PLOS ONE 11, e0163962 (2016).

18. Martins, A. L., Walavalkar, N. M., Anderson, W. D., Zang, C. \& Guertin, M. J. Universal correction of enzymatic sequence bias reveals molecular signatures of protein/dna interactions. Nucleic acids research 46, e9 (2018).

19. Daley, T. \& Smith, A. D. Modeling genome coverage in single-cell sequencing. Bioinformatics 30, 3159-3165 (2014).

20. Rougvie, A. E. \& Lis, J. T. The RNA polymerase ii molecule at the 5' end of the uninduced hsp70 gene of d. Melanogaster is transcriptionally engaged. Cell 54, 795-804 (1988).

21. Core, L. J. et al. Defining the status of RNA polymerase at promoters. Cell reports 2, 1025-1035 (2012).

22. Furey, T. S. ChIPseq and beyond: New and improved methodologies to detect and characterize proteinDNA interactions. Nature Reviews Genetics 13, 840852 (2012).

23. Love, M. I., Huber, W. \& Anders, S. Moderated estimation of fold change and dispersion for RNA-seq data with DESeq2. Genome Biology 15, 550 (2014).

24. Martin, M. Cutadapt removes adapter sequences from high-throughput sequencing reads. EMBnet.journal 17, 10-12 (2011). 
25. Oliphant, T. E. A guide to numpy. 1, (Trelgol Publishing USA, 2006).

26. McKinney, W. \& others. Data structures for statistical computing in python. in Proceedings of the 9th python in science conference 445, 51-56 (Austin, TX, 2010).

27. Stolarczyk, M., Reuter, V. P., Smith, J. P., Magee, N. E. \& Sheffield, N. C. Refgenie: A reference genome resource manager. GigaScience 9, (2020).

28. Quinlan, A. R. BEDTools: The swiss-army tool for genome feature analysis: BEDTools: The swiss-army tool for genome feature analysis. Current Protocols in Bioinformatics 47, 11.12.1-11.12.34 (2014).

29. Kent, W. J., Zweig, A. S., Barber, G., Hinrichs, A. S. \& Karolchik, D. BigWig and bigbed: Enabling browsing of large distributed datasets. Bioinformatics (Oxford, England) 26, 2204-2207 (2010).

30. Langmead, B. \& Salzberg, S. L. Fast gapped-read alignment with bowtie 2. Nat. Methods 9, 357-359 (2012).

31. Edwards, R. \& Edwards, J. A. Fastq-pair: Efficient synchronization of paired-end fastq files. BioRxiv 552885 (2019).

32. Magoc, T. \& Salzberg, S. L. FLASH: Fast length adjustment of short reads to improve genome assemblies. Bioinformatics 27, 2957-2963 (2011).

33. Li, H. \& Durbin, R. Fast and accurate short read alignment with burrows-wheeler transform. Bioinformatics 25, 1754-60 (2009).

34. Kurtz, S., Narechania, A., Stein, J. C. \& Ware, D. A new method to compute k-mer frequencies and its application to annotate large repetitive plant genomes. $B M C$ genomics 9, 517 (2008). 
bioRxiv preprint doi: https://doi.org/10.1101/2020.02.27.956110; this version posted July 15, 2020. The copyright holder for this preprint (which

was not certified by peer review) is the author/funder, who has granted bioRxiv a license to display the preprint in perpetuity. It is made available under aCC-BY 4.0 International license.

\section{Supplemental text}

\section{Gene counts table}

PEPPRO provides a project level counts table that simplifies downstream analyses. Here, we import the PEPPRO project counts table and construct a DESeq data set in a few lines of code.

\# 1. Load the PEPPRO R package.

library (PEPPROr)

\# 2. Load the PEP project using the project configuration file.

prj = Project ("peppro_paper $\cdot$ yaml")

\# 3. Load the project gene counts table.

counts $=$ read.csv $($ file.path $($ paste $(\operatorname{config}(\operatorname{prj})$ \$metadata\$output_dir, "/summary/PEPPRO_countData.csv")))

\# 4. Only keep the H9 untreated or H9 HDAC inhibitor treated samples.

counts = counts [,c("geneName", "H9_PRO-seq_1", "H9_PRO-seq_2", "H9_PRO-seq_3",

"H9_treated_PRO-seq_1", "H9_treated_PRO-seq_2",

"H9_treated_PRO-seq_3")]

\# 5. Convert the counts table to a matrix by removing the gene name column.

count_matrix = as.matrix (counts [,-"geneName"])

\# 6. Set the rownames of the matrix object to be the gene names.

rownames (count_matrix) = counts\$geneName

\# 7. Create a data.frame that defines the sample information.

coldata $=$ data.frame $(\operatorname{condition}=c(\operatorname{rep}($ untreated", 3), rep("treated", 3)) $)$

\# 8. Set the rownames of the sample information data.frame to match the counts matrix.

rownames $($ coldata $)=$ colnames (count_matrix)

\# 9. Load the DESeq2 package.

library ("DESeq2")

\# 10. Create a DESeq data set from our counts matrix and the sample information data.frame.

$\mathrm{dds}=$ DESeqDataSetFromMatrix (countData = count_matrix,

colData $=$ coldata,

design $=\sim$ condition) 
bioRxiv preprint doi: https://doi.org/10.1101/2020.02.27.956110; this version posted July 15, 2020. The copyright holder for this preprint (which was not certified by peer review) is the author/funder, who has granted bioRxiv a license to display the preprint in perpetuity. It is made available under aCC-BY 4.0 International license.

\section{Supplemental figures}

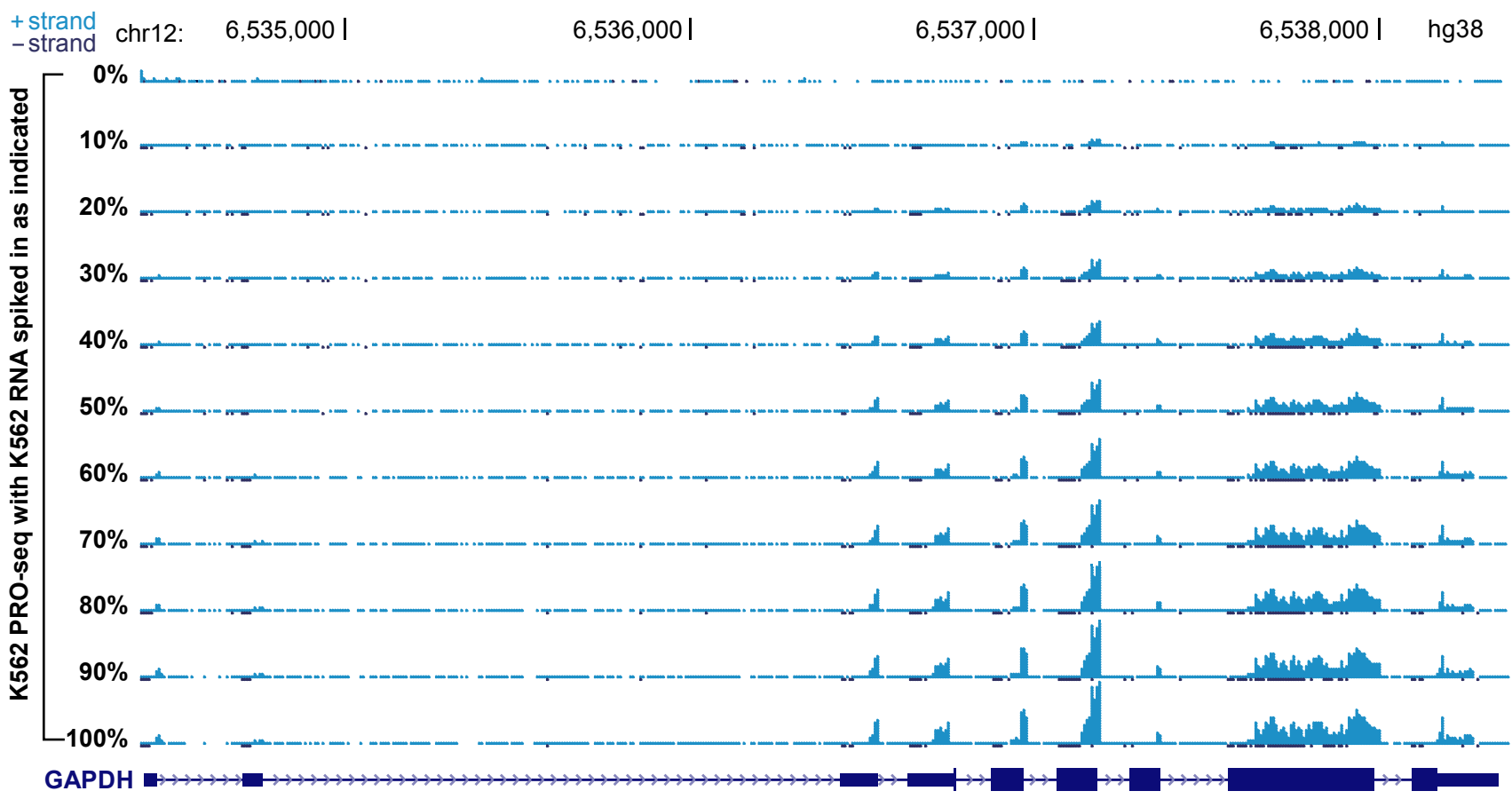

Fig. S1: K562 RNA-seq spike-in signal tracks show increasing exonic coverage. GAPDH exonic coverage is enriched as the percentage of RNA-seq reads increases, and is visualized particularly well at exons 6 and 8. Each sample library is composed of 70M total reads.

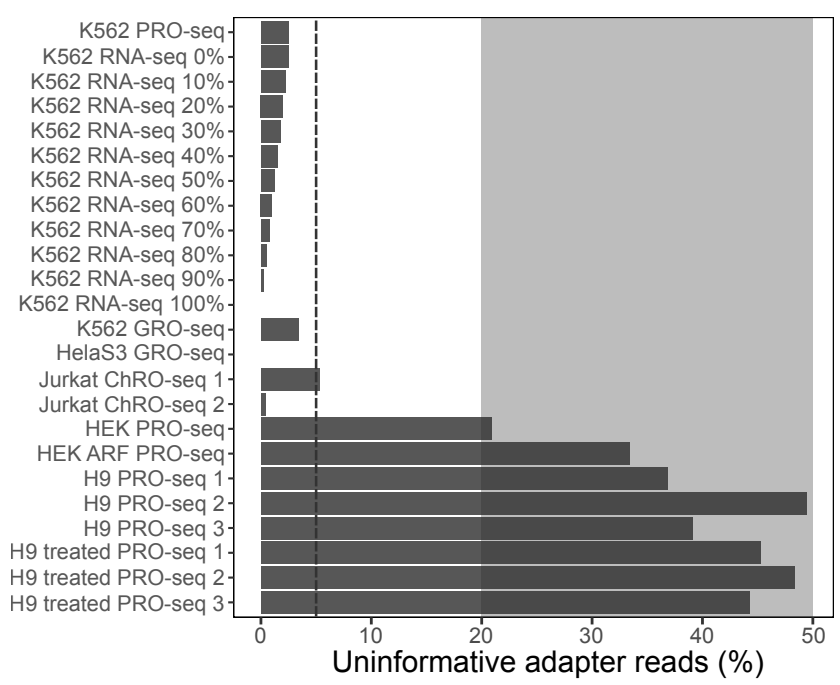

Fig. S2: Percentage of uninformative adapter reads following adapter removal for test set samples. The HEK and H9 libraries contain more adapter-adapter reads because PAGE-mediated size selection was excluded from the protocol (Values below the dashed line are generally recommended for PAGE-purified libraries. Shaded region represents the recommended abundance of adapter reads for libraries without PAGE purification.) 
bioRxiv preprint doi: https://doi.org/10.1101/2020.02.27.956110; this version posted July 15,2020 . The copyright holder for this preprint (which was not certified by peer review) is the author/funder, who has granted bioRxiv a license to display the preprint in perpetuity. It is made available under aCC-BY 4.0 International license.

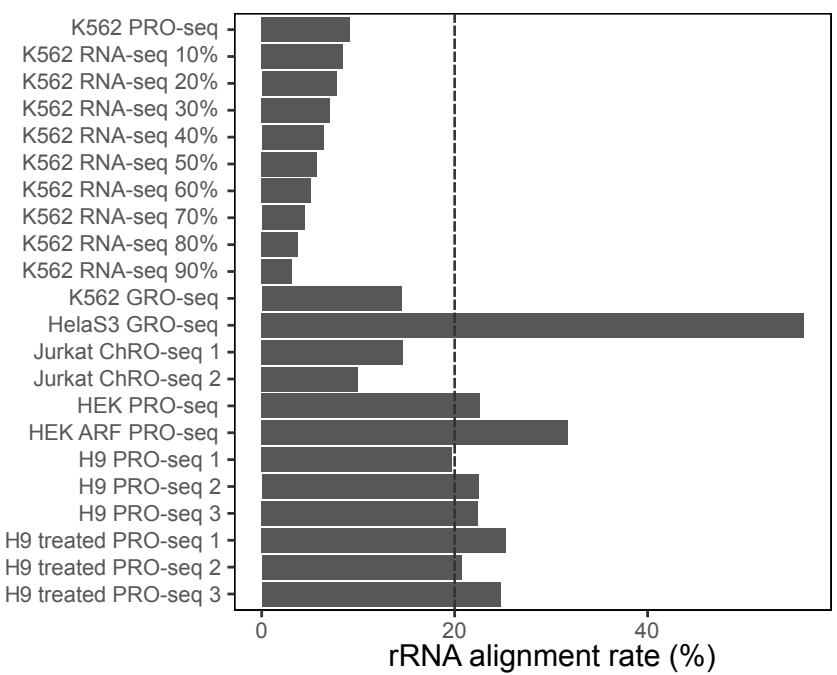

Fig. S3: Ribosomal DNA alignment rates for test set samples. The HelaS3 GRO-seq sample is highly enriched for ribosomal RNA transcripts compared to other test samples. Values below the dashed line are recommended.

A

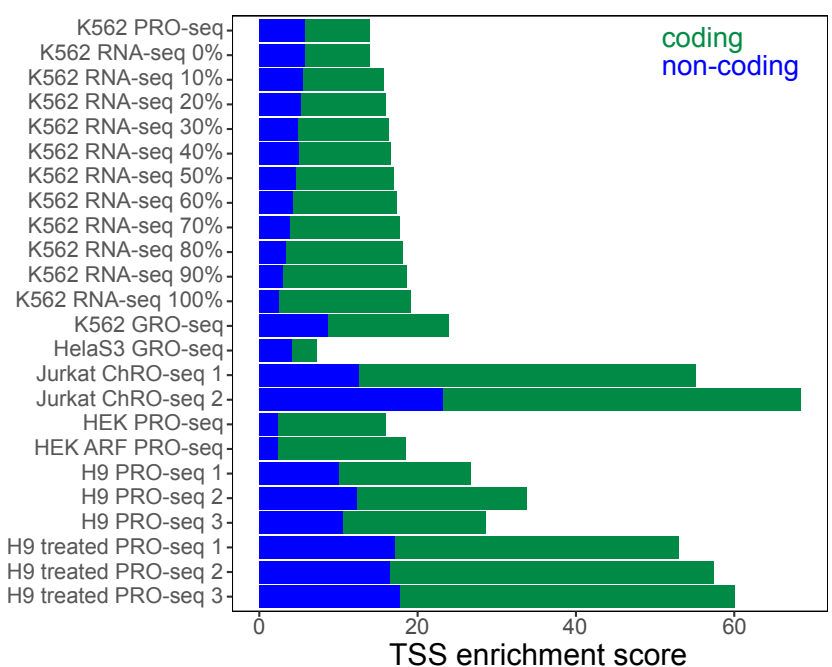

B

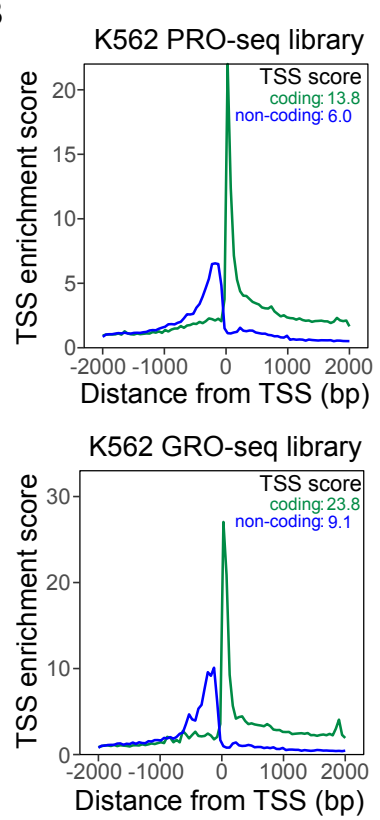

C

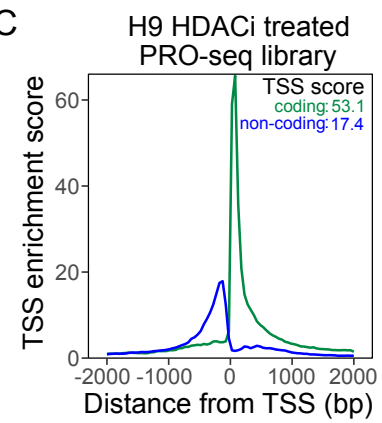

E

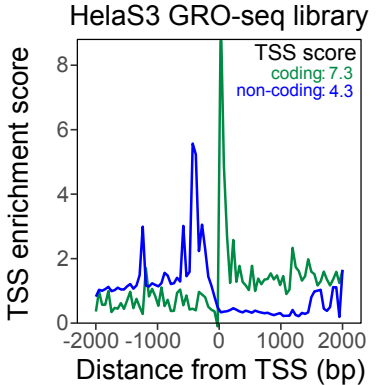

Fig. S4: TSS enrichment. A) TSS enrichment scores for test set samples. B) Representative high-quality PRO-seq TSS enrichment plot. C) TSS enrichment plot in romidepsin treated PRO-seq library. D) Representative high quality GRO-seq TSS enrichment plot E) Representative example of lower quality GRO-seq TSS enrichment plot.

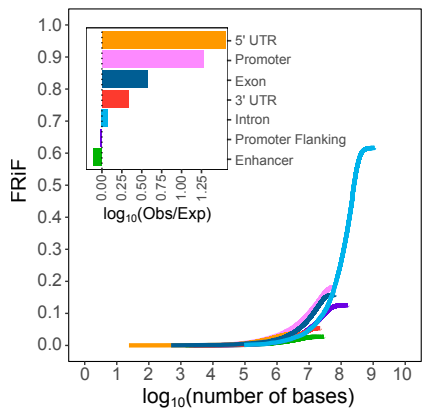

Fig. S5: Fraction of Reads in Features in ChRO-seq. Cumulative FRiF and FRiF (inset) plots for example Jurkat ChRO-seq 1 library test sample shows increased enrichment of promoter sequences. 
bioRxiv preprint doi: https://doi.org/10.1101/2020.02.27.956110; this version posted July 15, 2020. The copyright holder for this preprint (which

was not certified by peer review) is the author/funder, who has granted bioRxiv a license to display the preprint in perpetuity. It is made available under aCC-BY 4.0 International license.

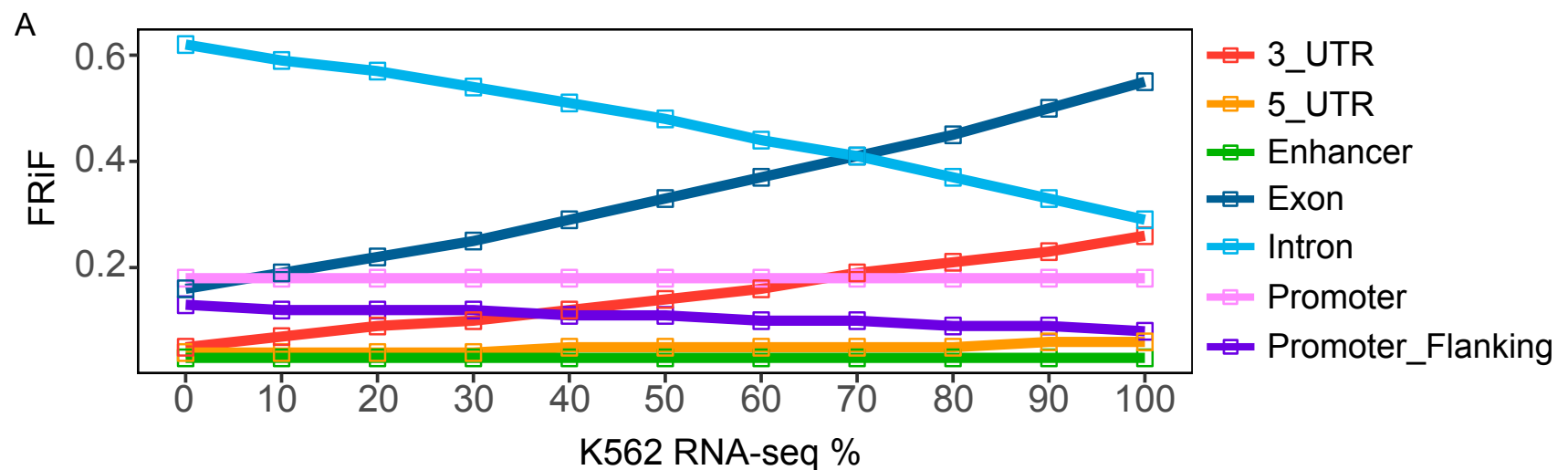

B
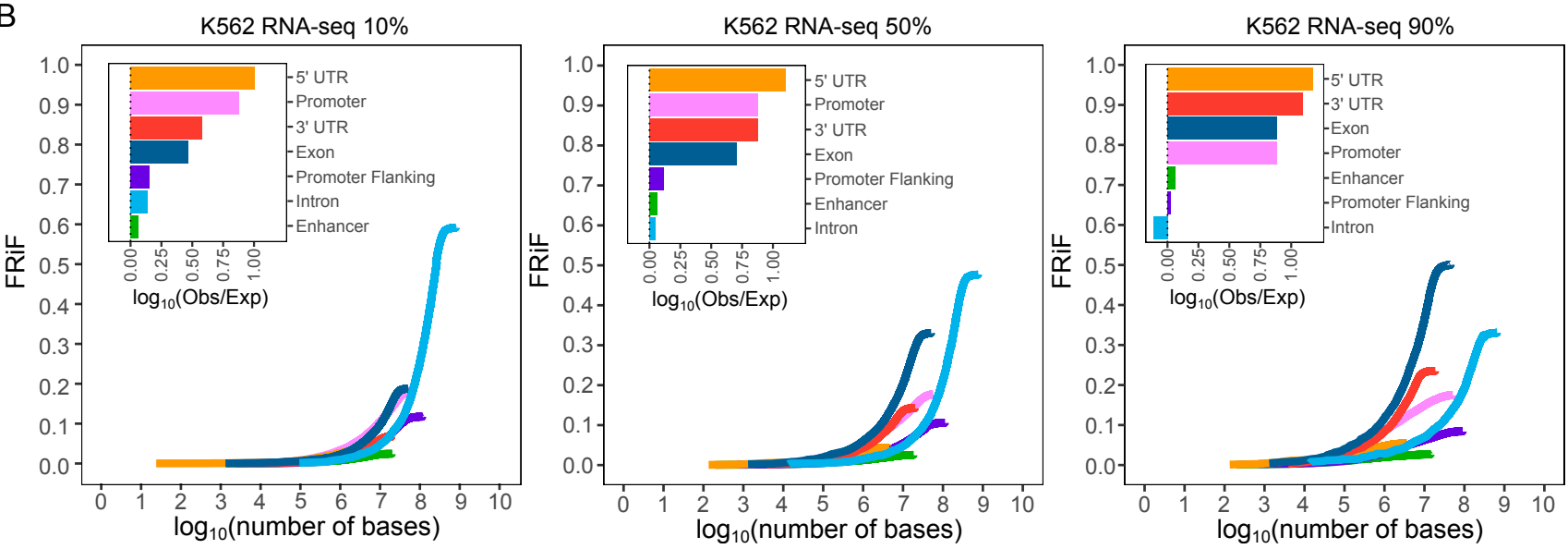

Fig. S6: RNA-seq spike-in shows how FRiF changes with mRNA contamination. A) Increasing percentages of RNA-seq spike-in lead to changes in the fraction of reads in features (FRiF). B) Cumulative FRiF plots at 10\%, 50\%, and 90\% RNA-seq spike-in. Plot insets represent the expected versus observed fraction of reads in genomic features. Each spike-in library contains 70 million total reads. 
bioRxiv preprint doi: https://doi.org/10.1101/2020.02.27.956110; this version posted July 15, 2020. The copyright holder for this preprint (which was not certified by peer review) is the author/funder, who has granted bioRxiv a license to display the preprint in perpetuity. It is made available under aCC-BY 4.0 International license.

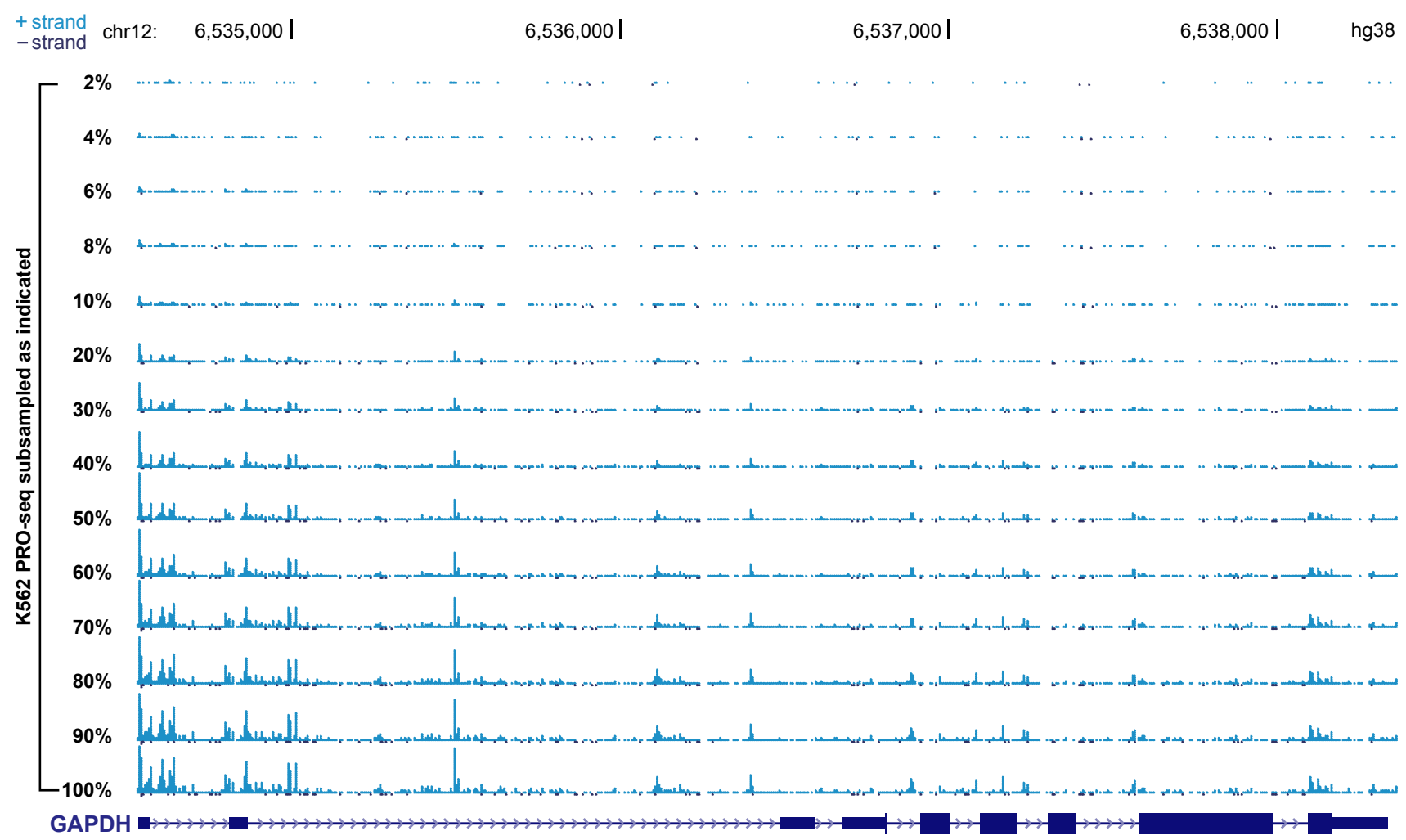

Fig. S7: K562 PRO-seq signal tracks show increasing coverage with depth. Incrementally subsampled K562 PRO-seq library signal tracks display reduced relative coverage at a representative locus (GAPDH).

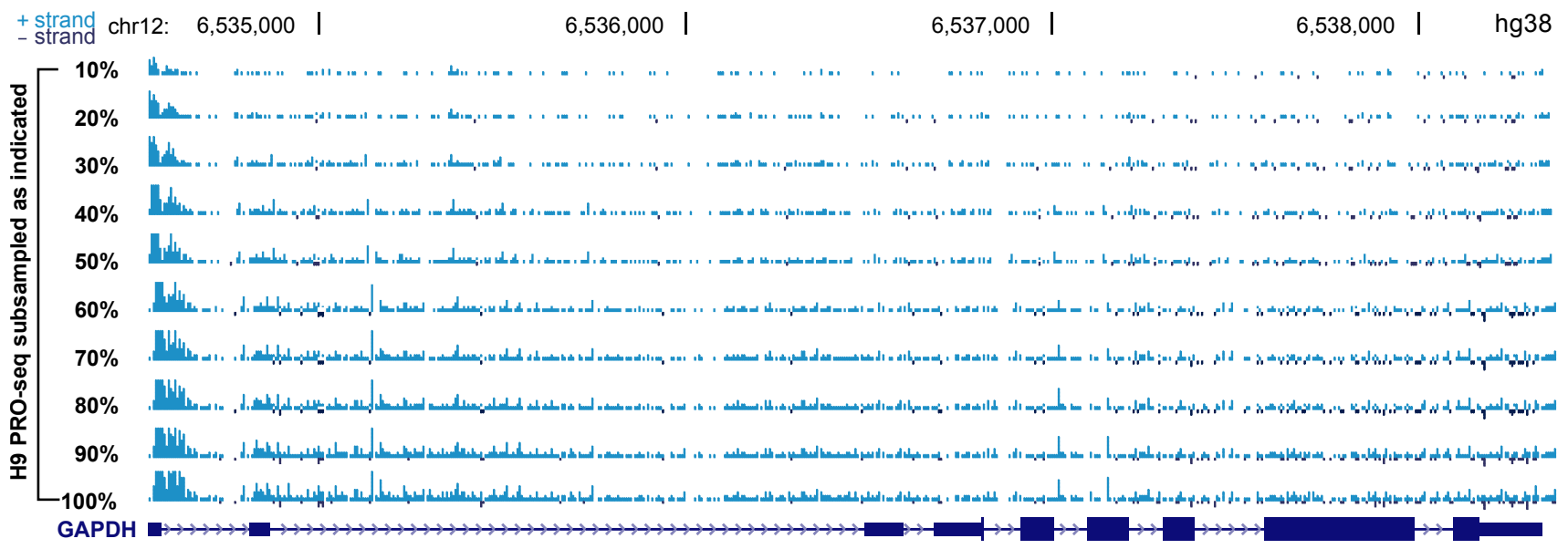

Fig. S8: H9 PRO-seq 2 signal tracks show increasing coverage with depth. Incrementally subsampled H9 PRO-seq 2 library signal tracks display reduced relative coverage at a representative locus (GAPDH). 
bioRxiv preprint doi: https://doi.org/10.1101/2020.02.27.956110; this version posted July 15,2020 . The copyright holder for this preprint (which was not certified by peer review) is the author/funder, who has granted bioRxiv a license to display the preprint in perpetuity. It is made available under aCC-BY 4.0 International license.

A

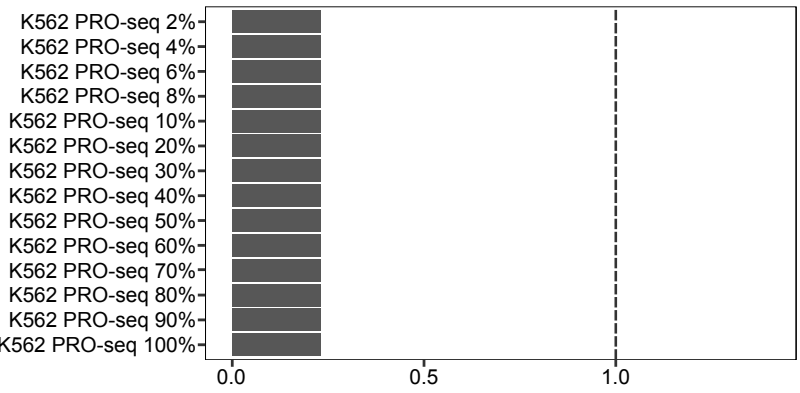

C

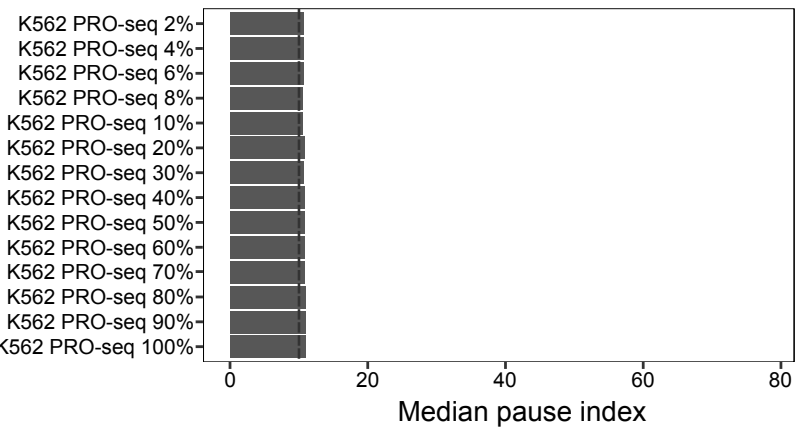

E

K562 PRO-seq 2\%K562 PRO-seq 4\% K562 PRO-seq 6\% K562 PRO-seq 8\% K562 PRO-seq 10\% K562 PRO-seq 20\% K562 PRO-seq $30 \%$ K562 PRO-seq 40\% K562 PRO-seq 50\% K562 PRO-seq 60\% K562 PRO-seq 70\% K562 PRO-seq 80\% K562 PRO-seq $90 \%$ K562 PRO-seq 100\%

G

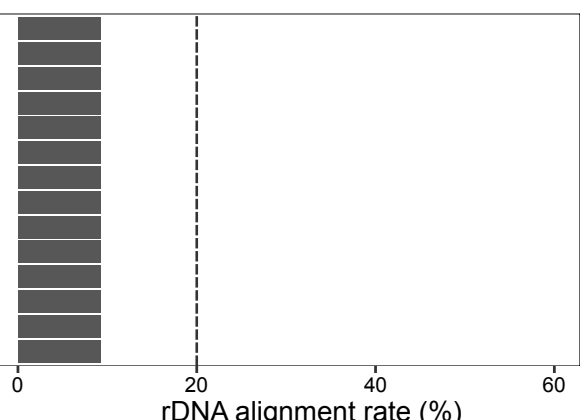

B

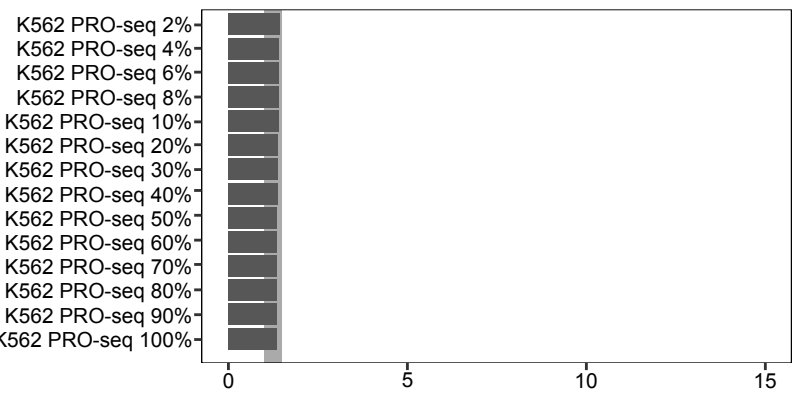

D

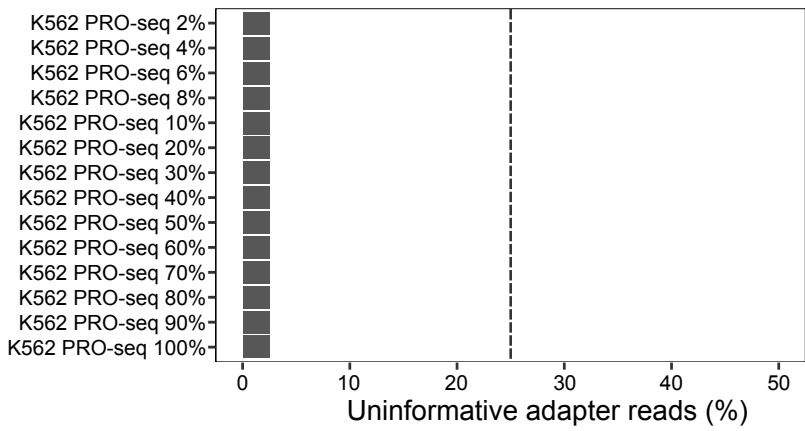

F

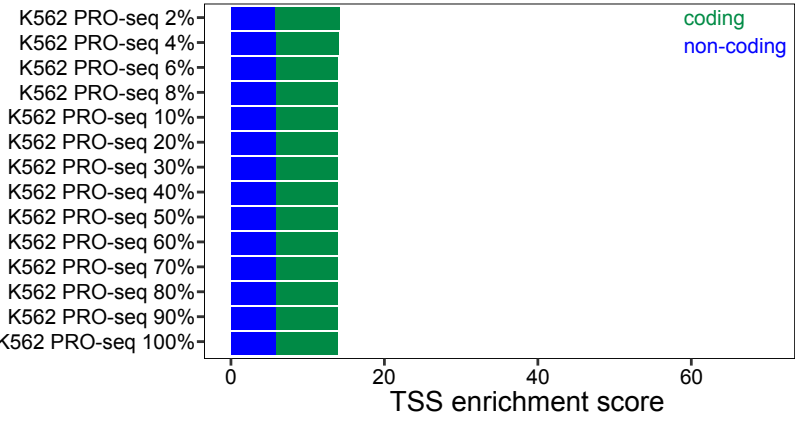

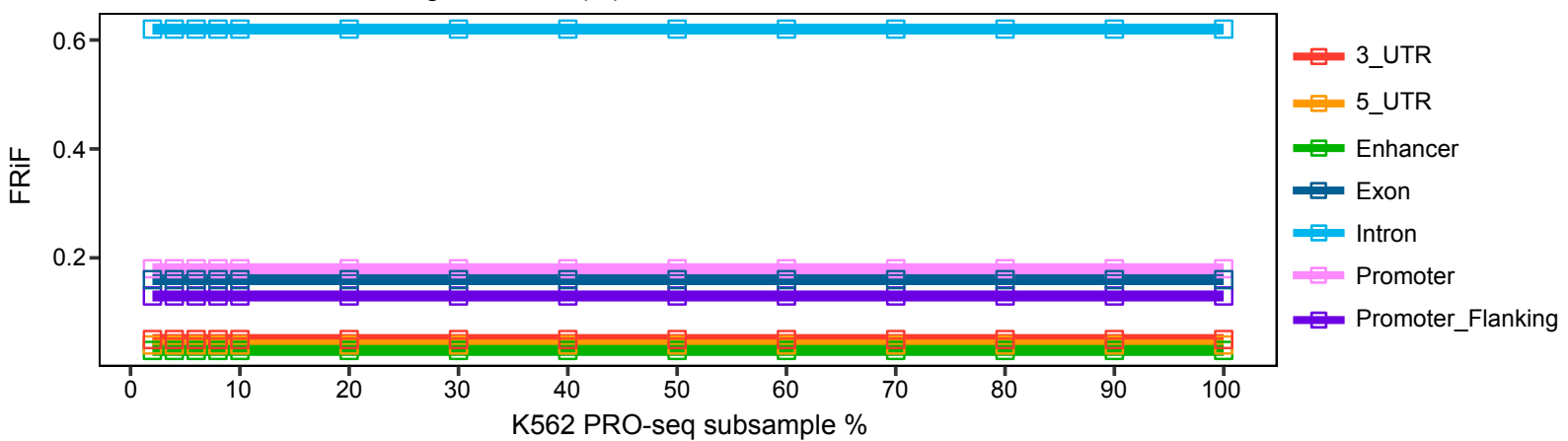

Fig. S9: QC metrics are not affected by sequencing depth in subsampled K562 PRO-seq. Using subsampled K562 PRO-seq data, we show how various metrics behave across a spectrum of sequencing depths: A) Degradation ratio, B) mRNA contamination, C) Pause index, D) the percentage of uninformative adapter reads, E) the rDNA alignment rate, F) and the TSS enrichment scores are unaffected by sequencing depth. G) The FRiF and cumulative FRiF is unaffected by sequencing depth. The complete K562 PRO-seq library (100\%) contains approximately 497 million reads. 
bioRxiv preprint doi: https://doi.org/10.1101/2020.02.27.956110; this version posted July 15, 2020. The copyright holder for this preprint (which was not certified by peer review) is the author/funder, who has granted bioRxiv a license to display the preprint in perpetuity. It is made available under aCC-BY 4.0 International license.

A

E

G

$\mathrm{H}$

C

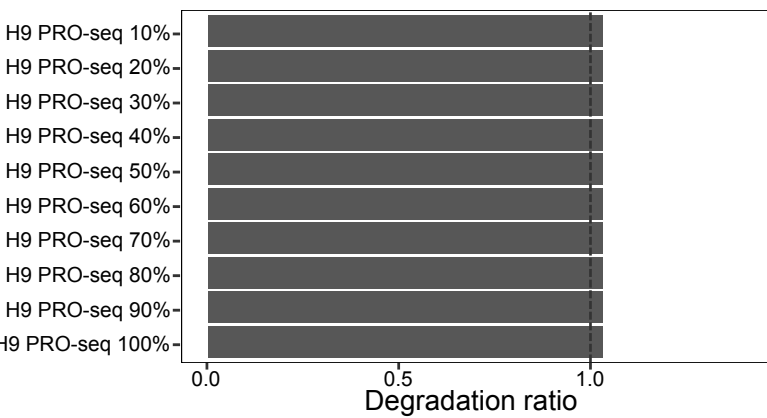

H9 PRO-seq $10 \%-$ H9 PRO-seq $20 \%$ H9 PRO-seq $30 \%$ H9 PRO-seq $40 \%$ H9 PRO-seq $50 \%$ H9 PRO-seq $60 \%$ H9 PRO-seq $70 \%$ H9 PRO-seq $80 \%$ H9 PRO-seq $90 \%$ H9 PRO-seq $100 \%$

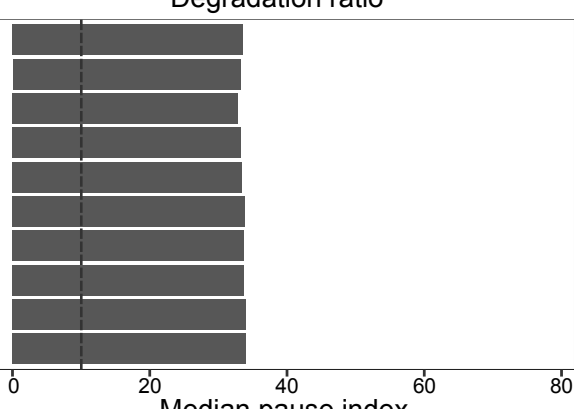

H9 PRO-seq 10\% H9 PRO-seq $20 \%$ H9 PRO-seq $30 \%$ H9 PRO-seq $40 \%$ H9 PRO-seq $50 \%$ H9 PRO-seq $60 \%$ H9 PRO-seq $70 \%$ H9 PRO-seq $80 \%$ H9 PRO-seq $90 \%$ H9 PRO-seq $100 \%$ -

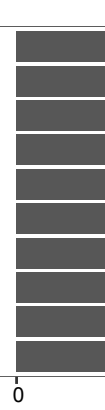

Median pause index
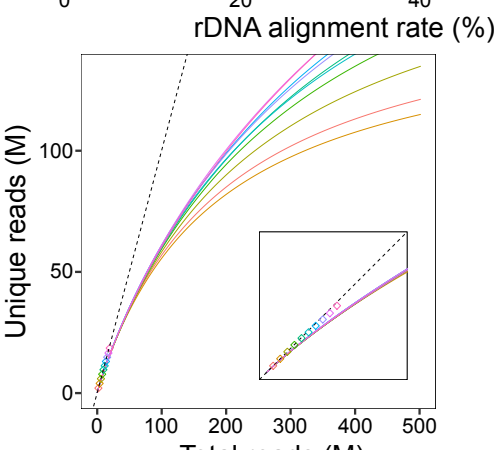

Total reads $(\mathrm{M})$
B

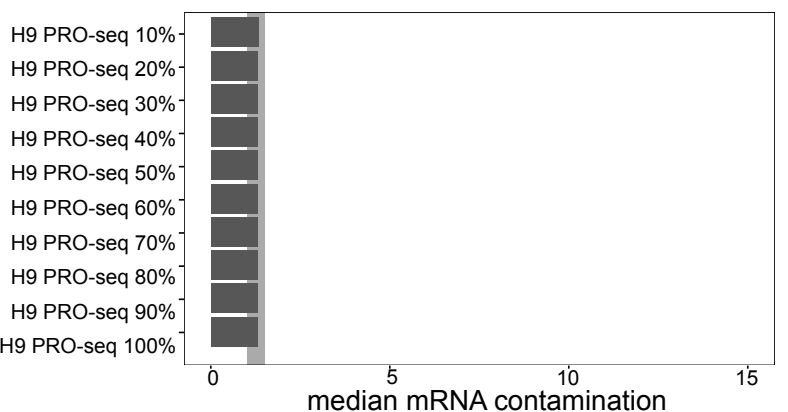

D

H9 PRO-seq $10 \%$ H9 PRO-seq $20 \%$ H9 PRO-seq $30 \%$ H9 PRO-seq $40 \%$ H9 PRO-seq $50 \%$ H9 PRO-seq $60 \%-$ H9 PRO-seq $70 \%$ H9 PRO-seq $80 \%$ H9 PRO-seq $90 \%$ H9 PRO-seq $100 \%$

\section{$\mathrm{F}$}
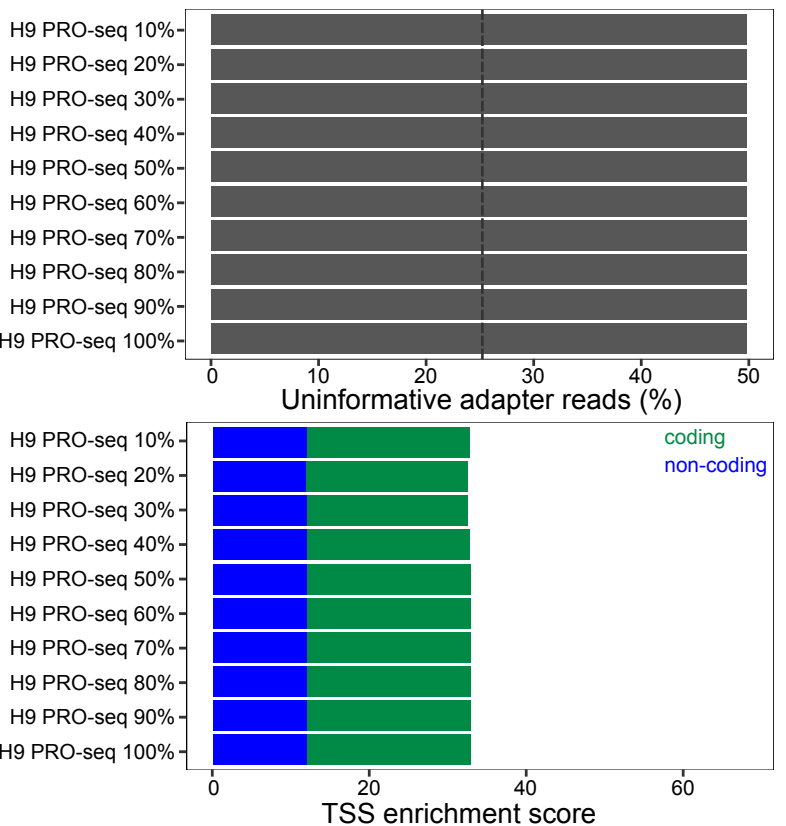

TSS enrichment score

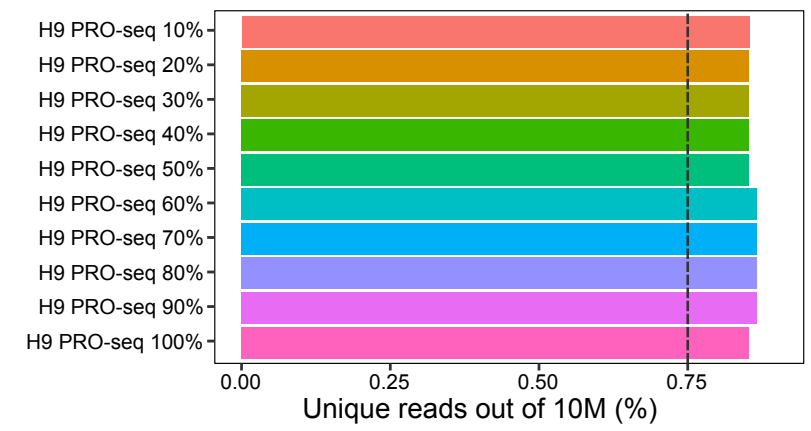

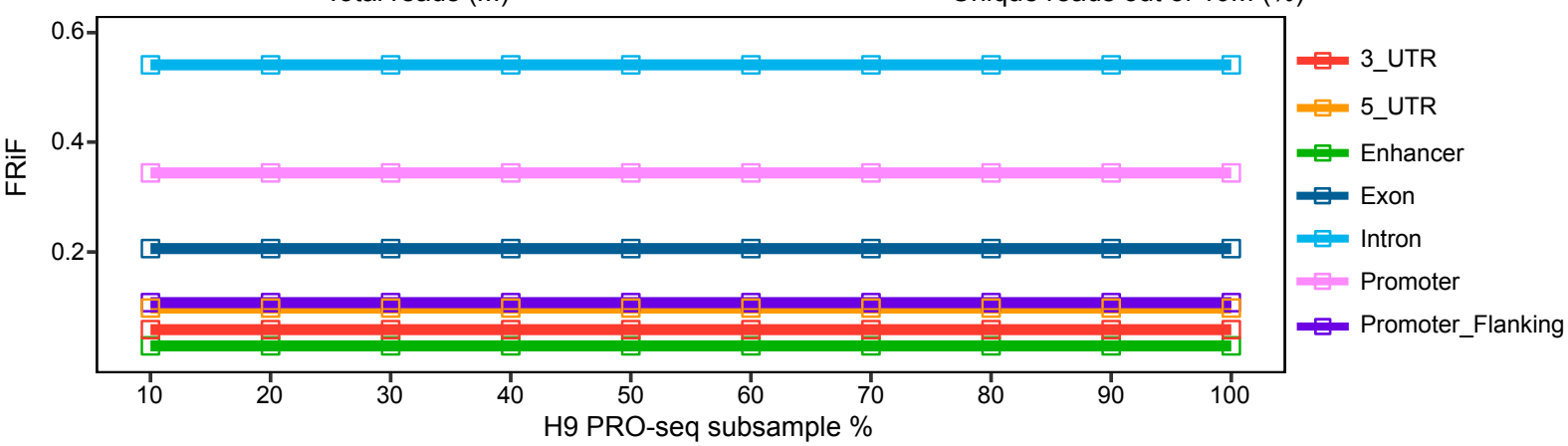

Fig. S10: QC metrics are not affected by sequencing depth in subsampled H9 PRO-seq. Using subsampled H9 PRO-seq data, we show how various metrics behave across a spectrum of sequencing depths: A) Degradation ratio, B) mRNA contamination, C) Pause index, $D$ ) the percentage of uninformative adapter reads, $E$ ) the rDNA alignment rate, $F$ ) and the TSS enrichment scores are unaffected by sequencing depth. G) Library complexity traces plot the read count versus externally calculated deduplicated read counts. Deduplication is a prerequisite, so these plots may only be produced for samples with UMIs. Inset zooms to region from 0 to double the maximum number of unique reads. The position of curves in the left panel at a sequencing depth of 10 million reads (dashed line represents minimum recommended percentage of unique reads). H) The FRiF and cumulative FRiF is unaffected by sequencing depth. The complete H9 PRO-seq library (100\%) contains approximately 116 million reads. 
bioRxiv preprint doi: https://doi.org/10.1101/2020.02.27.956110; this version posted July 15, 2020. The copyright holder for this preprint (which was not certified by peer review) is the author/funder, who has granted bioRxiv a license to display the preprint in perpetuity. It is made available under aCC-BY 4.0 International license.

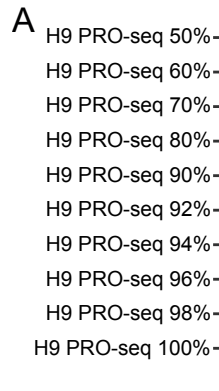

C

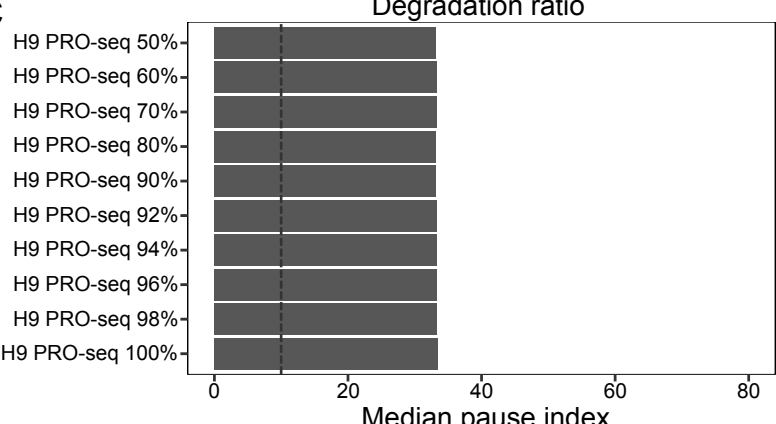

$\mathrm{E}$

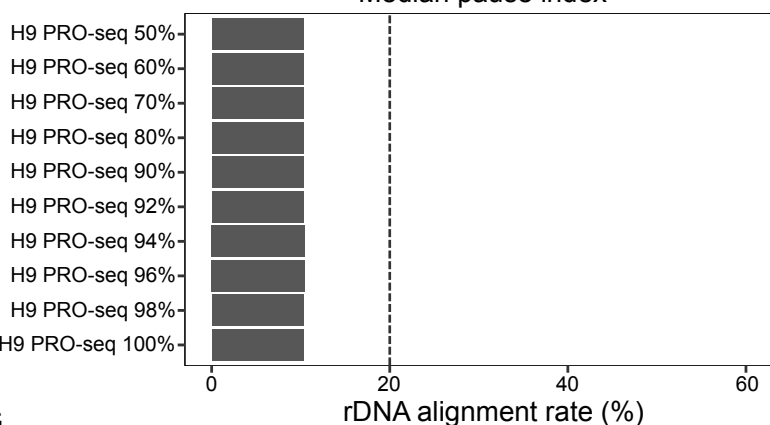

G

$\mathrm{H}$

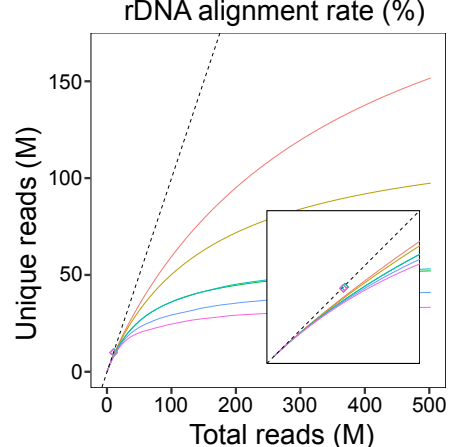

B

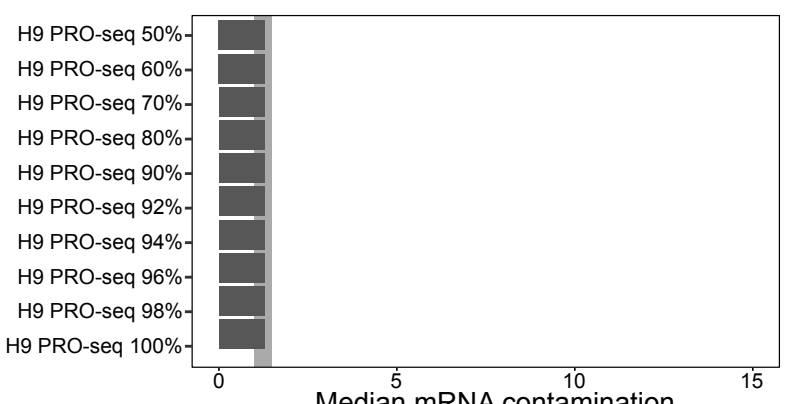

D

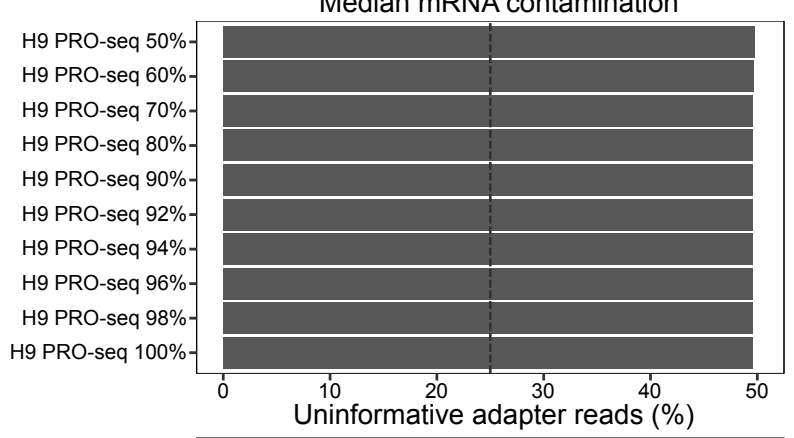

$\mathrm{F}$
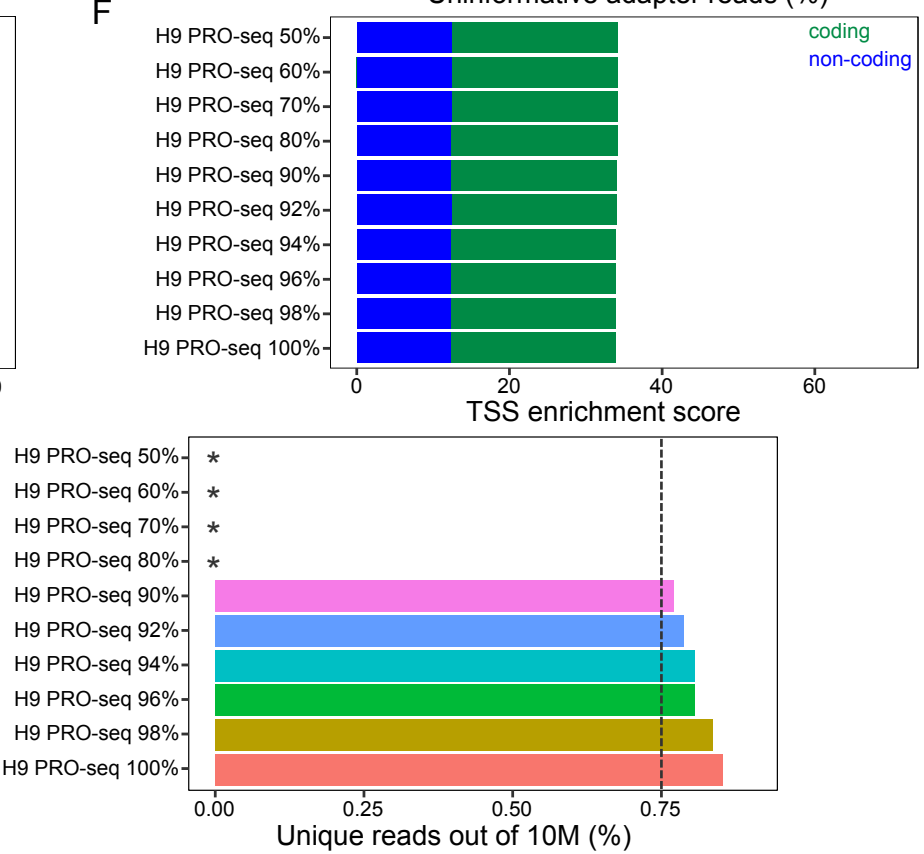

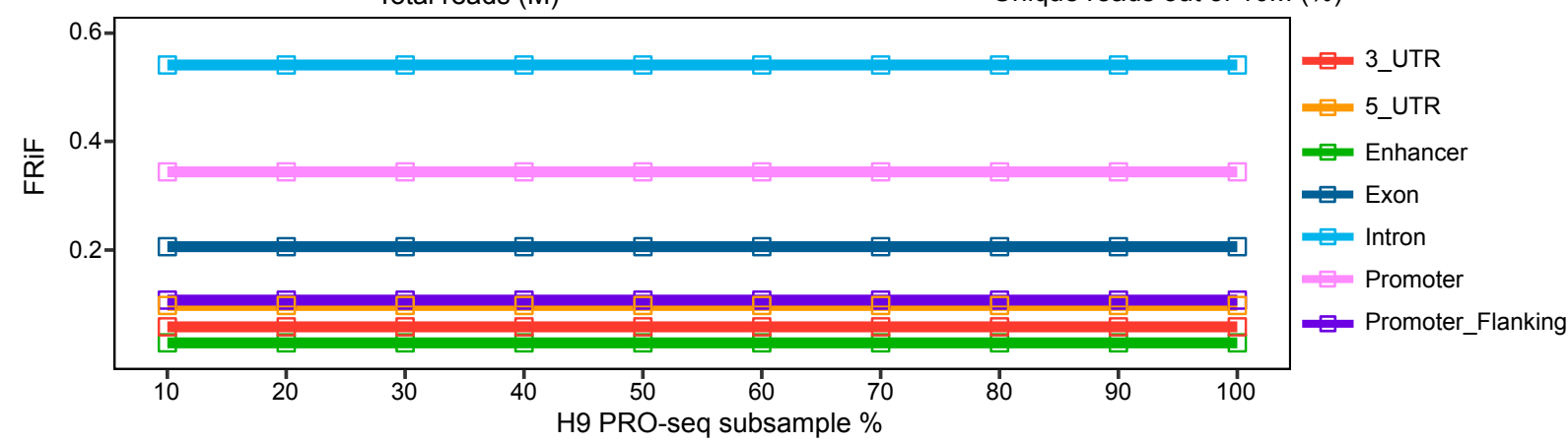

Fig. S11: Low library complexity does not affect QC metrics. Using a synthetic set of libraries, we show how various metrics behave across a spectrum of complexity: A) Degradation ratio, B) mRNA contamination, C) Pause index, D) the percentage of uninformative adapter reads, E) the rDNA alignment rate, F) and the TSS enrichment scores are unaffected by low complexity. G) Library complexity traces plot the read count versus externally calculated deduplicated read counts. Deduplication is a prerequisite, so these plots may only be produced for samples with UMIs. Inset zooms to region from 0 to double the maximum number of unique reads. The right panel represents the position of curves in the left panel at a sequencing depth of 10 million reads (dashed line represents minimum recommended percentage of unique reads). "Libraries with less than 90\% uniqueness could not be extrapolated due to saturation. H) The FRiF and cumulative FRiF is unaffected by low complexity. Each library contains 30 million total reads. 
bioRxiv preprint doi: https://doi.org/10.1101/2020.02.27.956110; this version posted July 15, 2020. The copyright holder for this preprint (which was not certified by peer review) is the author/funder, who has granted bioRxiv a license to display the preprint in perpetuity. It is made available under aCC-BY 4.0 International license.
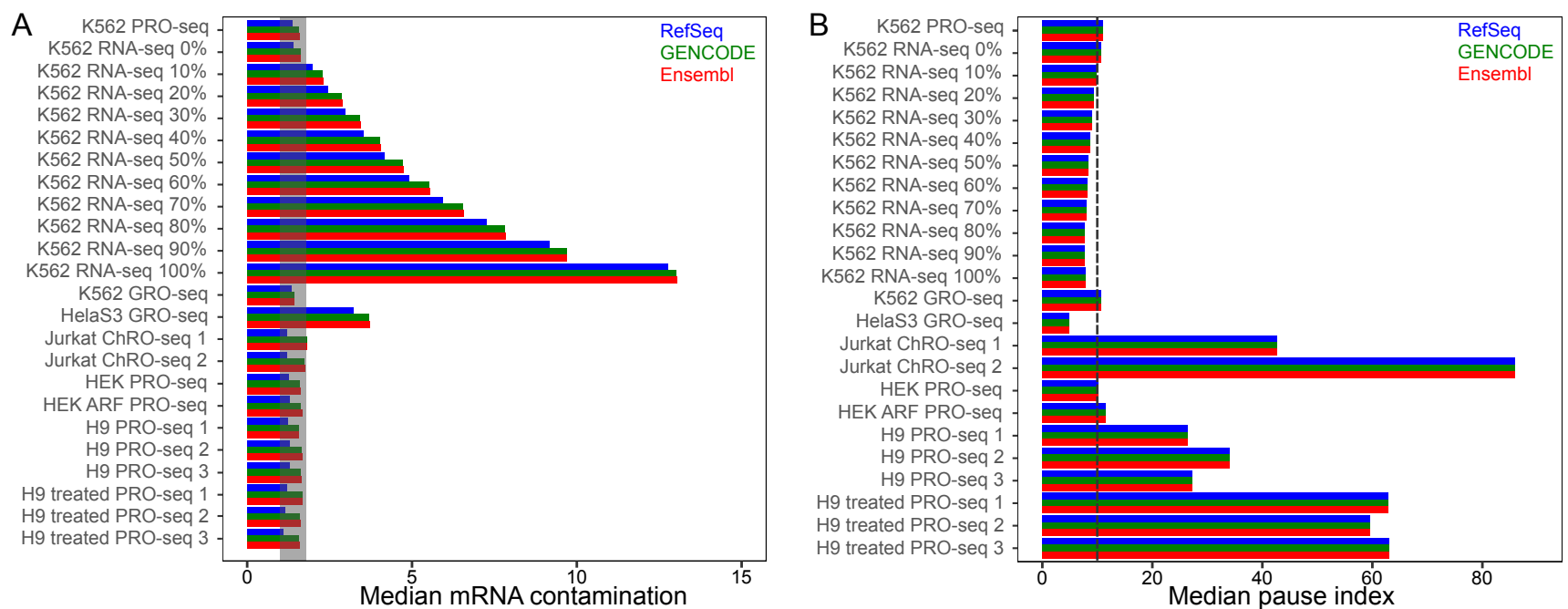

Fig. S12: Alternate annotation sources do not affect mRNA contamination and pause index. A) The mRNA contamination metric and $B$ ) the pause index metric are robust across annotations. 